\title{
BOUNDARY-INITIATED WAVE PHENOMENA IN THERMOELASTIC MATERIALS
}

\author{
BY \\ T. S. ÖNCÜ AND T. B. MOODIE \\ University of Alberta, Edmonton, Alberta, Canada
}

Summary. The linear theory of Gurtin and Pipkin, and Chen and Gurtin is adopted to study one-dimensional progressive waves generated by thermal and mechanical disturbances applied at the boundary of a circular hole in an unbounded homogeneous thermoelastic medium. A ray-series approach is employed to generate asymptotic wavefront expansions for the field variables. The characteristics of the propagation process are obtained simply and directly. The solution is then specialized to the case where this theory reduces to the linearized theory of Lord and Shulman, and numerical results for various values of material parameters obtained from the ray-series solution in conjunction with the use of Pade approximants are displayed graphically.

1. Introduction. The paradox of instantaneous propagation of thermal disturbances intrinsic to the classical theory of heat conduction in rigid materials has motivated many researchers to seek an alternative to the classical theory. Consequently, a number of theories free from this paradox have been proposed in recent years [1-6]. Almost all of these theories have also been extended to include deformable materials [7-13]. In the present paper we shall be concerned with only one of these theories. An extensive list of many others is given in Sawatzky and Moodie [14].

Taking a departure from the fundamental concepts developed by Coleman and Noll [15] and by Coleman [16], Gurtin and Pipkin [4] established their general theory of heat conduction in rigid materials with memory in 1968. In 1970, Chen and Gurtin [9] extended this theory to include deformable materials with memory. The theory of Gurtin and Pipkin and of Chen and Gurtin implies two finite speeds of propagation for thermomechanical waves in such materials. These speeds are usually referred to as the first and second sound speeds. The first sound speed is quasimechanical and lies near the acoustical speed for the material whereas the second sound speed is associated with a quasi-thermal wave. Shear waves, which do not generate volume changes, remain unaffected by thermomechanical coupling. This result is in agreement with that of the thermomechanical theories based on the classical theory of heat conduction.

In this paper we study the propagation of one-dimensional progressive waves emanating from the boundary of a circular hole of radius $a$ in an unbounded homoge-

Received June 1, 1989.

(C)1990 Brown University 
neous isotropic thermoelastic plate by invoking the linearized theory of Gurtin and Pipkin and of Chen and Gurtin. We generate asymptotic wavefront expansions using a ray-series approach which has been employed by Moodie and Tait [17] for rigid, Sawatzky and Moodie [14] for elastic, and McCarthy et al. [18] for viscoelastic heat conductors. After deriving the explicit expressions for the sound speeds we find that when $C_{t} / D_{c}<1$, where $C_{t}$ is the speed of purely thermal and $C_{d}$ the speed of purely elastic dilatation waves for the material, the faster and slower speeds are the first and second sounds speeds, whereas for $C_{t} / C_{d} \geq 1$ the slower and faster speeds are the first and second sound speeds, respectively. A similar result has been obtained by Achenbach [19], who considered only materials whose heat conduction is governed by the Maxwell-Cattaneo relation, for the cases $C_{t} / C_{d}<(1+\gamma)^{1 / 2}$ and $C_{t} / C_{d}>(1+\gamma)^{1 / 2}$ where $\gamma$ is the thermoelastic coupling constant. Our result shows that the first and second sound speeds can be determined independently from $\gamma$. The ray-series method employed here not only enables us to determine the propagation characteristics of thermoelastic disturbances but also provides an efficient algorithm for numerical computations. Moreover, the range of validity of these computations can be extended to larger domains of physical interest with the aid of Pade approximants. Various Padé-extended numerical results for a relatively simple example are also included in this paper.

2. Formulation. Consider an unbounded homogeneous isotropic thermoelastic plate which occupies the region $0<a<r<+\infty$ in a plane polar coordinate system $r, \varphi$. Initially the plate is undistorted, at rest and in thermal equilibrium with a uniform absolute temperature $T_{0}$. In any departure from this thermodynamic equilibrium the field variables are assumed to be functions of time $t$ and radial coordinate $r$ only. It is further assumed that departures from the equilibrium are small, that is, the displacement gradient and the relative temperature change are small at all times when compared to unity. That is,

$$
\sqrt{\left(\frac{\partial u_{r}}{\partial r}\right)^{2}+\left(\frac{\partial u_{\varphi}}{\partial r}\right)^{2}} \ll 1 \text { and }\left|T-T_{0}\right| \ll T_{0},
$$

for all $t$, where $T(r, t)$ is the absolute temperature, and $u_{r}(r, t)$ and $u_{\varphi}(r, t)$ are the radial and angular displacements, respectively. As a result of the above assumptions the linearized equations of linear momentum and energy in the absence of body forces and external heat sources are

$$
\begin{aligned}
\rho_{0} \frac{\partial^{2} u_{r}}{\partial t^{2}} & =\frac{\partial \sigma_{r r}}{\partial r}+\frac{1}{r}\left(\sigma_{r r}-\sigma_{\varphi \varphi}\right), \\
\rho_{0} \frac{\partial^{2} u_{\varphi}}{\partial t^{2}} & =\frac{\partial \sigma_{r \varphi}}{\partial r}+\frac{2}{r} \sigma_{r \varphi}, \\
\rho_{0} \frac{\partial e}{\partial t} & =\operatorname{tr}\left\{\boldsymbol{\sigma} \frac{\partial \boldsymbol{\varepsilon}}{\partial t}\right\}-\nabla \cdot \mathbf{q},
\end{aligned}
$$

where $\rho_{0}$ is the uniform mass density of the initial equilibrium, $\sigma_{r r}, \sigma_{r \varphi}$, and $\sigma_{\varphi \varphi}$ are the plane polar components of the symmetric stress tensor $\sigma(r, t), e(r, t)$ is the 
specific internal energy, $\mathbf{q}(r, t)$ is the heat flux and $\boldsymbol{\varepsilon}(r, t)$ is the strain tensor with the plane polar components

$$
\varepsilon_{r r}=\frac{\partial u_{r}}{\partial r}, \quad \varepsilon_{r \varphi}=\varepsilon_{\varphi r}=\frac{1}{2}\left(\frac{\partial u_{\varphi}}{\partial r}-\frac{u_{\varphi}}{r}\right), \quad \varepsilon_{\varphi \varphi}=\frac{u_{r}}{r} .
$$

In Eq. (2.4), $\nabla$ is the gradient in the appropriate coordinate system and $\operatorname{tr}$ denotes the trace. We also introduce the specific entropy $\eta(r, t)$, the specific free energy

$$
\psi(r, t)=e-T \eta,
$$

and the temperature gradient

$$
\mathbf{g}(r, t)=\nabla T
$$

for future reference.

For the model to be completed, we assume that the present values of $\psi=\psi(r, t)$, $\boldsymbol{\sigma}=\boldsymbol{\sigma}(r, t), \mathbf{q}=\mathbf{q}(r, t), \eta=\eta(r, t)$, and $e=e(r, t)$ are given by the response functionals $\Psi, \Sigma, \mathbf{Q}, N$, and $E$ as

$$
\begin{gathered}
\psi=\Psi\left(\Lambda^{t}\right), \quad \boldsymbol{\sigma}=\mathbf{\Sigma}\left(\Lambda^{t}\right), \quad \mathbf{q}=\mathbf{Q}\left(\Lambda^{t}\right), \\
\eta=N\left(\Lambda^{t}\right) \quad \text { and } \quad e=E\left(\Lambda^{t}\right),
\end{gathered}
$$

where $E$ is connected to $\Psi$ and $N$ through the relation

$$
E=\Psi+T N,
$$

and the thermal history array $\Lambda^{t}$ for the thermoelastic plate is assumed as

$$
\Lambda^{t}=\left(\varepsilon, T, \bar{T}^{t}, \overline{\mathbf{g}}^{t}\right) \text {. }
$$

In identity (2.10), $\bar{T}^{t}$ and $\overline{\mathbf{g}}^{t}$ are the summed histories of absolute temperature and temperature gradient at $r$ up to time $t$ which are defined as

$$
\bar{T}^{t}(r, s)=\int_{0}^{s} T(r, t-\lambda) d \lambda \text { and } \overline{\mathbf{g}}^{t}(r, s)=\int_{0}^{s} \mathbf{g}(r, t-\lambda) d \lambda .
$$

If the array $(\varepsilon, T, \mathbf{g})$ and the response functionals (2.8) satisfy the hypothesis of the theorem of Chen and Gurtin [9], then the free energy functional $\Psi$ determines $\Sigma$, $N$, and $\mathbf{Q}$ from the relations

$$
\begin{aligned}
& \Sigma=\rho_{0} \frac{\partial \Psi}{\partial \boldsymbol{\varepsilon}}, \\
& N=-\frac{\partial \Psi}{\partial T},
\end{aligned}
$$

and

$$
\mathbf{Q} \cdot \mathbf{h}=-\rho_{0} T\left[\frac{d}{d z} \psi\left(\boldsymbol{\varepsilon}, T, \bar{T}^{t}, \overline{\mathbf{g}}^{t}+z \mathbf{h}\right)\right]_{t=0},
$$

where $\mathbf{h}$ is any constant nonzero vector consistent with the hypothesis of the theorem. The components of the partial derivative of $\Psi$ with respect to the strain tensor $\varepsilon$ are

$$
\left(\frac{\partial \Psi}{\partial \varepsilon}\right)_{x_{i} x_{j}}=\frac{\partial \Psi}{\partial \varepsilon_{x_{j} x_{i}}}, \quad i, j=1,2
$$


where $x_{1}=r$ and $x_{2}=\varphi$. Following Gurtin and Pipkin [4] and Chen and Gurtin [9], we then choose the free energy functional $\Psi$ as follows:

$$
\begin{aligned}
\Psi\left(\Lambda^{t}\right)= & \frac{1}{\rho_{0}} \hat{\psi}(\boldsymbol{\varepsilon}, T)-\int_{0}^{\infty} \beta^{\prime}(s) \bar{T}^{t}(r, s) \\
& +\frac{1}{2 \rho_{0} T \alpha(0)}\left[\int_{0}^{\infty} \alpha^{\prime}(s) \overline{\mathbf{g}}^{t}(r, s) d s\right] \cdot\left[\int_{0}^{\infty} \alpha^{\prime}(s) \overline{\mathbf{g}}^{t}(r, s) d s\right],
\end{aligned}
$$

where $\alpha(s)$ is the thermal relaxation function, $\beta(s)$ the energy relaxation function, $\alpha^{\prime}(s)$ and $\beta^{\prime}(s)$ their respective derivatives, and $\alpha(0)$ the instantaneous conductivity. We further specialize the function $\hat{\psi}(\boldsymbol{\varepsilon}, t)$ to be the free energy function of the classical theory of thermoelasticity. For isotropic materials $\hat{\psi}(\boldsymbol{\varepsilon}, t)$ takes the form [20]

$$
\hat{\psi}(\boldsymbol{\varepsilon}, T)=\rho_{0} \hat{\psi}_{0}+\mu \operatorname{tr}\{\boldsymbol{\varepsilon} \boldsymbol{\varepsilon}\}+\frac{\lambda}{2}(\operatorname{tr} \boldsymbol{\varepsilon})^{2}-(3 \lambda+2 \mu) \alpha_{t}\left(T-T_{0}\right) \operatorname{tr} \boldsymbol{\varepsilon}-\frac{\rho_{0} c}{2 T_{0}}\left(T-T_{0}\right)^{2},
$$

where $\lambda$ and $\mu$ are the isothermal Lamé constants, $\alpha_{t}$ the coefficient of linear thermal expansion, $c$ the specific heat at constant volume, and $\hat{\psi}_{0}=\Psi\left(\mathbf{0}, T_{0}, \bar{T}_{0}^{t}, \overline{\mathbf{0}}^{t}\right)$ the specific free energy in the initial equilibrium state. With the above choice of the free energy functional, the stress tensor, specific internal energy, and heat flux obtained from Eqs. (2.9) and (2.12)-(2.14) are

$$
\begin{gathered}
\sigma_{r r}=(2 \mu+\lambda) \varepsilon_{r r}+\lambda \varepsilon_{\varphi \varphi}-(3 \lambda+2 \mu) \alpha_{t} \theta, \\
\sigma_{r \varphi}=2 \mu \varepsilon_{r \varphi}, \\
\sigma_{\varphi \varphi}=(2 \mu+\lambda) \varepsilon_{\varphi \varphi}+\lambda \varepsilon_{r r}-(3 \lambda+2 \mu) \alpha_{t} \theta, \\
e=e_{0}+\frac{1}{\rho_{0}}(3 \lambda+2 \mu) \alpha_{t} T_{0}\left(\varepsilon_{r r}+\varepsilon_{\varphi \varphi}\right)+c \theta-\int_{0}^{\infty} \beta^{\prime}(s) \bar{\theta}^{t}(r, s) d s, \\
\mathbf{q}=\int_{0}^{\infty} \alpha^{\prime}(s) \overline{\mathbf{g}}^{t}(r, s) d s,
\end{gathered}
$$

where

$$
\theta(r, t)=T-T_{0},
$$

is the temperature difference and $e_{0}=E\left(\mathbf{0}, T_{0}, \bar{T}_{0}^{t}, \overline{\mathbf{0}}^{t}\right)$ is the specific energy of the initial equilibrium state. We omitted the second-order terms in $\boldsymbol{\varepsilon}, \theta$, and $\overline{\mathbf{g}}^{t}$ from the above constitutive equations. If we further impose the conditions

$$
\lim _{s \rightarrow+\infty} s^{2} \alpha(s)<\infty, \quad \lim _{s \rightarrow+\infty} s^{2} \beta(s)<\infty,
$$

the integrals in (2.21) and (2.22) may be integrated by parts [4] to yield

$$
\begin{gathered}
e=e_{0}+\frac{1}{\rho_{0}}(3 \lambda+2 \mu) \alpha_{t} T_{0}\left(\varepsilon_{r r}+\varepsilon_{\varphi \varphi}\right)+c \theta+\int_{0}^{\infty} \beta(s) \theta(r, t-s) d s, \\
\mathbf{q}=-\int_{0}^{\infty} \alpha(s) \nabla \theta(r, t-s) d s .
\end{gathered}
$$


We note that Fourier's law of heat conduction is not a special case of (2.26). However, if we choose the heat flux relaxation function $\alpha(s)$ as

$$
\alpha(s)=\frac{\kappa}{\tau} e^{-s / \tau}, \quad \tau>0,
$$

where $\kappa$ is the coefficient of thermal conductivity and $\tau$ is the thermal relaxation time, then (2.26) reduces to the Maxwell-Cattaneo relation

$$
\tau \frac{\partial \mathbf{q}}{\partial t}+\mathbf{q}=-\kappa \nabla \theta
$$

In this case, if we also assume that the free-energy functional is independent of the summed history of the absolute temperature or, equivalently,

$$
\beta(s)=0,
$$

then the present constitutive equations reduce to the constitutive equations of the linear theory of Lord and Shulman [7].

Upon substitution of the constitutive relation (2.18)-(2.20) and (2.25), (2.26) into the field equations, together with the use of strain-displacement relations (2.5), we arrive at the following thermoelastic equations

$$
\begin{gathered}
\frac{\partial^{2} u_{r}}{\partial r^{2}}+\frac{1}{r} \frac{\partial u_{r}}{\partial r}-\frac{u_{r}}{r^{2}}-\frac{1}{C_{d}^{2}} \frac{\partial^{2} u}{\partial t^{2}}=\frac{(3 \lambda+2 \mu)}{(2 \mu+\lambda)} \alpha_{t} \frac{\partial \theta}{\partial r}, \\
\rho_{0} c \frac{\partial \theta}{\partial t}+(3 \lambda+2 \mu) \alpha_{t} T_{0} \frac{\partial}{\partial t}\left[\frac{\partial u_{r}}{\partial r}+\frac{u_{r}}{r}\right]+\int_{0}^{\infty} \rho_{0} \beta(s) \frac{\partial \theta}{\partial t}(r, t-s) d s \\
=\int_{0}^{\infty} \alpha(s)\left[\frac{\partial^{2}}{\partial r}+\frac{1}{r} \frac{\partial}{\partial r}\right] \theta(r, t-s) d s,
\end{gathered}
$$

and the shear equation

$$
\frac{\partial^{2} u_{\varphi}}{\partial r^{2}}+\frac{1}{r} \frac{\partial u_{\varphi}}{\partial r}-\frac{u_{\varphi}}{r^{2}}-\frac{1}{C_{s}^{2}} \frac{\partial^{2} u_{\varphi}}{\partial t^{2}}=0,
$$

where

$$
C_{d}=\left[\frac{2 \mu+\lambda}{\rho_{0}}\right]^{1 / 2}
$$

is the isothermal velocity of dilatational waves and

$$
C_{s}=\left[\frac{\mu}{\rho_{0}}\right]^{1 / 2}
$$

is the velocity of shear waves. In (2.31) the term $\operatorname{tr}\left\{\boldsymbol{\sigma} \frac{\partial \boldsymbol{\varepsilon}}{\partial t}\right\}$ has been neglected since it gives rise to second-order terms only. The above equations reveal that shear waves which produce no volume changes are independent from thermal effects. Thus, the theory of Gurtin and Pipkin and of Chen and Gurtin agrees with the classical theory of thermoelasticity in this regard.

In the present paper we restrict our attention to the waves generated by suddenly applied uniform temperature change and uniform surface tractions at the boundary 
of the circular hole. Consequently, we specify the initial and the boundary conditions as

$$
\begin{array}{r}
\theta(r, t)=\frac{\partial \theta}{\partial t}(r, t)=u_{r}(r, t)=\frac{\partial u_{r}}{\partial t}(r, t)=u_{\varphi}(r, t)=\frac{\partial u_{\varphi}}{\partial t}(r, t)=0, \\
a<r<+\infty,-\infty<t \leq 0, \\
\theta(a, t)=\theta_{1}(t), \quad \sigma_{r r}(a, t)=\sigma_{1}(t), \quad \sigma_{r \varphi}(a, t)=\sigma_{2}(t) \quad 0<t<+\infty .
\end{array}
$$

We shall assume that the thermal relaxation function $\alpha(t)$ and the energy relaxation function $\beta(t)$ have well-defined Taylor series expansions at $t=0$. Therefore,

$$
\begin{aligned}
& \alpha(t)=H(t) \sum_{i=0}^{\infty} \alpha_{i} \frac{t^{i}}{i !}, \\
& \beta(t)=H(t) \sum_{i=0}^{\infty} \beta_{i}^{0} \frac{t^{i}}{i !},
\end{aligned}
$$

where

$$
\alpha_{i}^{0}=\left.\frac{d^{i} \alpha}{d t^{i}}\right|_{t=0}, \quad \beta_{i}^{0}=\left.\frac{d^{i} \beta}{d t^{i}}\right|_{t=0},
$$

are the relaxation coefficients and $H(t)$ is the Heaviside step function. It suffices to specify the relaxation functions only near the time origin as the changes at the wavefronts are completely determined by the behaviour of these functions there [17]. Chen and Nunziato [21] have shown that for the second law of thermodynamics to be satisfied, $\alpha$ and $\beta$ should obey the restrictions

$$
\alpha_{0}^{0} \geq 0, \quad \alpha_{1}^{0} \leq 0, \quad \beta_{0}^{0} \geq 0, \quad \int_{0}^{\infty} \alpha(s) d s \geq 0 .
$$

We further impose the condition

$$
\alpha_{0}^{0}>0
$$

to assure well-defined finite discontinuity wavefronts propagating at finite speeds.

Prior to further study of the problem we introduce the following nondimensional quantities:

$$
\begin{gathered}
\hat{r}=\frac{r}{a}, \quad(\hat{t}, \hat{s}, \hat{\tau})=\frac{\kappa}{a^{2} \rho_{0} c}(t, s, \tau) \\
\hat{\theta}=\frac{\theta}{T_{0}}, \quad\left(\hat{u}_{r}, \hat{u}_{\varphi}\right)=\frac{2 \mu+\lambda}{(3 \lambda+2 \mu) \alpha_{t} T_{0}}\left(\frac{u_{r}}{a}, \frac{u_{\varphi}}{a}\right) \\
\left(\hat{\sigma}_{r r}, \hat{\sigma}_{r \varphi}, \hat{\sigma}_{\varphi \varphi}\right)=\frac{\left(\sigma_{r r}, \sigma_{r \varphi}, \sigma_{\varphi \varphi}\right)}{(3 \lambda+2 \mu) \alpha_{t} T_{0}}, \quad\left(\widehat{C}_{d}, \widehat{C}_{s}\right)=\frac{a \rho_{0} c}{\kappa}\left(C_{d}, C_{s}\right) \\
\hat{\alpha}=\frac{a^{2} \rho_{0} c}{\kappa^{2}} \alpha, \quad \hat{\beta}=\frac{a^{2} \rho_{0}}{\kappa} \beta .
\end{gathered}
$$

Henceforward, we use these nondimensional quantities but drop the carets to avoid notational complications. 
3. Ray-series solution. Assuming the radial displacement $u_{r}$ is generated by the scalar potential $\Phi(r, t)$ as

$$
u_{r}=\frac{\partial \Phi}{\partial r}
$$

the nondimensional equations associated with thermoelastic waves obtained from (2.30), (2.31), (2.42), and (3.1) are

$$
\begin{gathered}
\frac{\partial^{2} \Phi}{\partial r^{2}}+\frac{1}{r} \frac{\partial \Phi}{\partial r}-\frac{1}{C_{d}^{2}} \frac{\partial^{2} \Phi}{\partial t^{2}}=\theta \\
\frac{\partial \theta}{\partial t}+\int_{0}^{\infty} \beta(s) \frac{\partial \theta}{\partial t}(r, t-s) d s+\gamma \frac{\partial}{\partial t}\left[\frac{\partial^{2} \Phi}{\partial r^{2}}+\frac{1}{r} \frac{\partial \Phi}{\partial r}\right] \\
=\int_{0}^{\infty} \alpha(s)\left[\frac{\partial^{2}}{\partial r^{2}}+\frac{1}{r} \frac{\partial}{\partial r}\right] \theta(r, t-s) d s,
\end{gathered}
$$

where

$$
\gamma=\frac{(3 \lambda+2 \mu)^{2} \alpha_{t}^{2} T_{0}}{\rho_{0} c(2 \mu+\lambda)}
$$

is the thermoelastic coupling constant. Upon combining Eqs. (3.2) and (3.3) we find that thermoelastic waves $\Phi, \theta$ satisfy the integro-partial differential equation

$$
L\{\Phi, \theta\}=0,
$$

where

$$
\begin{aligned}
L f= & \frac{\partial}{\partial t}\left[\frac{1}{C_{d}^{2}} \frac{\partial^{2}}{\partial t^{2}}-(1+\gamma)\left(\frac{\partial^{2}}{\partial r^{2}}+\frac{1}{r} \frac{\partial}{\partial r}\right)\right] f \\
& +\int_{0}^{\infty} \beta(s) \frac{\partial}{\partial t}\left[\frac{1}{C_{d}^{2}} \frac{\partial^{2}}{\partial t^{2}}-\left(\frac{\partial^{2}}{\partial r^{2}}+\frac{1}{r} \frac{\partial}{\partial r}\right)\right] f(r, t-s) d s \\
& -\int_{0}^{\infty} \alpha(s)\left(\frac{\partial^{2}}{\partial r^{2}}+\frac{1}{r} \frac{\partial}{\partial r}\right)\left[\frac{1}{C_{d}^{2}} \frac{\partial^{2}}{\partial t^{2}}-\left(\frac{\partial^{2}}{\partial r^{2}}+\frac{1}{r} \frac{\partial}{\partial r}\right)\right] f(r, t-s) d s .
\end{aligned}
$$

As a consequence of the assumption (3.1), the nondimensional constitutive equations of $\sigma_{r r}$ and $\sigma_{\varphi \varphi}$ become

$$
\begin{gathered}
\sigma_{r r}=\frac{\partial^{2} \Phi}{\partial r^{2}}+\frac{\nu}{r} \frac{\partial \Phi}{\partial r}-\theta, \\
\sigma_{\varphi \varphi}=\nu \frac{\partial^{2} \Phi}{\partial r^{2}}+\frac{1}{r} \frac{\partial \Phi}{\partial r}-\theta,
\end{gathered}
$$

where

$$
\nu=1-2 \frac{C_{s}^{2}}{C_{d}^{2}},
$$


and the initial and boundary conditions which generate the thermoelastic waves considered here can be written as

$$
\begin{gathered}
\theta(r, t)=\frac{\partial \theta}{\partial t}(r, t)=\Phi(r, t)=\frac{\partial \Phi}{\partial t}(r, t)=0, \quad 1<r<+\infty, \quad-\infty<t \leq 0, \\
\theta(1, t)=\theta_{1}(t), \quad \frac{\partial^{2} \Phi}{\partial r^{2}}+\left.\frac{\nu}{r} \frac{\partial \Phi}{\partial r}\right|_{r=1}=\sigma_{1}(t)+\theta_{1}(t), \quad 0<t<+\infty .
\end{gathered}
$$

After nondimensionalization, the shear equation remains identical to its dimensional counterpart and reads

$$
\frac{\partial^{2} u_{\varphi}}{\partial r^{2}}+\frac{1}{r} \frac{\partial u_{\varphi}}{\partial r}-\frac{u_{\varphi}}{r^{2}}-\frac{1}{C_{s}^{2}} \frac{\partial^{2} u_{\varphi}}{\partial t^{2}}=0,
$$

whereas the related initial and boundary conditions take the forms

$$
\begin{gathered}
u_{\varphi}(r, t)=\frac{\partial u_{\varphi}}{\partial t}(r, t)=0, \quad-\infty<t \leq 0, \quad 1 \leq r<+\infty, \\
\sigma_{r \varphi}(1 / t)=\sigma_{2}(t), \quad 0<t<+\infty,
\end{gathered}
$$

where the nondimensional constitutive equation for $\sigma_{r \varphi}$ is

$$
\sigma_{r \varphi}=\frac{C_{s}^{2}}{C_{d}^{2}}\left(\frac{\partial u_{\varphi}}{\partial r}-\frac{u_{\varphi}}{r}\right) .
$$

In this section we apply the ray-series method to solve the problems given by $(3.5)$ and (3.12) subject to conditions (3.10), (3.11) and (3.13), (3.14), respectively. Consequently, we represent the scalar potential $\Phi(r, t)$, the absolute temperature $\theta(r, t)$ and the angular displacement $u_{\varphi}(r, t)$ in terms of their asymptotic expansions

$$
\begin{aligned}
\Phi(r, t) & =\sum_{n=2}^{\infty} \phi_{n}(r) F_{n}(t-P(r)), & & \phi_{n} \equiv 0, n<2, \\
\theta(r, t) & =\sum_{n=0}^{\infty} T_{n}(r) F_{n}(t-P(r)), & & T_{n} \equiv 0, n<0, \\
u_{\varphi}(r, t) & =\sum_{n=1}^{\infty} U_{n}(r) F_{n}(t-S(r)), & & U_{n} \equiv 0, n<1,
\end{aligned}
$$

where the $F_{n}$ 's are related by

$$
F_{n}^{\prime}=F_{n-1}, \quad n=1,2, \ldots,
$$

with the prime denoting differentiation with respect to the entire argument. Equation (3.19) enables us to determine all of the $F_{n}$ 's from the waveform $F_{0}$ by successive integrations

We first solve the thermoelastic problem given by Eqs. (3.5), (3.10), and (3.11). The ray-series solution of the shear problem may be obtained in a similar fashion. To determine the coefficients $T_{n}(r)$ and the phase $P$, we substitute (3.17) into (3.5), employ the expressions for $\alpha$ and $\beta$ from (2.37) and (2.38) in the resulting equation 
and equate the coefficients of $F_{n-3}$. The terms involving integrals are evaluated by means of the formula

$$
\int_{0}^{\infty} \frac{s^{i}}{i !} F_{n}(t-s) d s=F_{n+1+i}(t), \quad i \geq 0
$$

which results from integration by parts. The result of the above manipulations is

$$
\begin{aligned}
\frac{1}{C_{d}^{2}} T_{n} & (1+\gamma)\left\{\left(P^{\prime}\right)^{2} T_{n}-P^{\prime}\left(2 T_{n-1}^{\prime}+\frac{1}{r} T_{n-1}\right)+T_{n-2}^{\prime \prime}+\frac{1}{r} T_{n-2}^{\prime}\right\} \\
= & {\left[\frac{\left(P^{\prime}\right)^{2}}{C_{d}^{2}}-\left(P^{\prime}\right)^{4}\right] \sum_{j=0}^{n} \alpha_{j}^{0} T_{n-j}+\left[2\left(P^{\prime}\right)^{3}-\frac{P^{\prime}}{C_{d}^{2}}\right] \sum_{j=0}^{n-1} \alpha_{j}^{0}\left(2 T_{n-j-1}^{\prime}+\frac{1}{r} T_{n-j-1}\right) } \\
& +\left[\frac{1}{C_{d}^{2}}-6\left(P^{\prime}\right)^{2}\right] \sum_{j=0}^{n-1} \alpha_{j}^{0}\left(T_{n-j-1}^{\prime \prime}+\frac{1}{r} T_{n-j-2}^{\prime}\right)+\left(P^{\prime}\right)^{2} \sum_{j=0}^{n-2} \alpha_{j}^{0} \frac{1}{r^{2}} T_{n-j-2} \\
& +P^{\prime} \sum_{j=0}^{n-3} \alpha_{j}^{0}\left[4 T_{n-j-4}^{\prime \prime \prime}+\frac{6}{r} T_{n-j-3}^{\prime \prime}-\frac{2}{r^{2}} T_{n-j-3}^{\prime}+\frac{1}{r^{3}} T_{n-j-3}\right] \\
& -\sum_{j=0}^{n-4} \alpha_{j}^{0}\left[T_{n-j-4}^{(i v)}+\frac{2}{r} T_{n-j-4}^{\prime \prime \prime}-\frac{1}{r^{2}} T_{n-j-4}^{\prime \prime}+\frac{1}{r^{3}} T_{n-j-2}^{\prime}\right] \\
& +\left[\left(P^{\prime}\right)^{2}-\frac{1}{C_{d}^{2}}\right]_{j=0}^{n-1} \beta_{j}^{0} T_{n-j-1}-P^{\prime} \sum_{j=0}^{n-2} \beta_{j}^{0}\left(2 T_{n-j-2}^{\prime}+\frac{1}{r}\right) \\
& +\sum_{j=0}^{n-3} \beta_{j}^{0}\left(T_{n-j-3}^{\prime \prime}+\frac{1}{r} T_{n-j-3}^{\prime}\right) \quad n=0,1,2, \ldots
\end{aligned}
$$

For the sake of brevity, we have omitted the terms involving $P^{\prime \prime}, P^{\prime \prime \prime}$, and $P^{(i v)}$ from (3.21) since it is shown later that $P^{\prime}$ is constant.

The first equation in the sequence (3.21), that is, the equation for $n=0$, is independent of the terms omitted and of the form

$$
\left[\alpha_{0}^{0}\left(P^{\prime}\right)^{4}-\left(1+\gamma+\frac{\alpha_{0}^{0}}{C_{d}^{2}}\right)\left(P^{\prime}\right)^{2}+\frac{1}{C_{d}^{2}}\right] T_{0}(r)=0 .
$$

This result proves that $P^{\prime}$ is independent of $r$ and, therefore, all higher order derivatives of $P$ vanish. Since we may require without loss of generality that $T_{0}(r) \neq 0$, (3.22) reduces to the eikonal equation

$$
\alpha_{0}^{0}\left(P^{\prime}\right)^{4}-\left(1+\gamma+\frac{\alpha_{0}^{0}}{C_{d}^{2}}\right)\left(P^{\prime}\right)^{2}+\frac{1}{C_{d}^{2}}=0,
$$

whose solution can be expressed as

$$
\left(P^{\prime}\right)^{2}=\frac{1}{2 \alpha_{0}^{0}}\left\{\left(1+\gamma+\frac{\alpha_{0}^{0}}{C_{d}^{2}}\right) \pm \Gamma^{1 / 2}\right\},
$$


where

$$
\Gamma=\left(\frac{\alpha_{0}^{0}}{C_{d}^{2}}+\gamma-1\right)^{2}+4 \gamma .
$$

Integrating the ordinary differential equation (3.24) along the ray associated with the thermoelastic waves we then obtain

$$
\begin{aligned}
& P(r)=\bar{P} \pm(r-1) P_{1}^{\prime}, \\
& P(r)=\bar{P} \pm(r-1) P_{2}^{\prime},
\end{aligned}
$$

where

$$
\begin{aligned}
& P_{1}^{\prime}=\left\{\frac{1}{2 \alpha_{0}^{0}}\left[\left(1+\gamma+\frac{\alpha_{0}^{0}}{C_{d}^{2}}\right)+\Gamma^{1 / 2}\right]\right\}^{1 / 2}, \\
& P_{2}^{\prime}=\left\{\frac{1}{2 \alpha_{0}^{0}}\left[\left(1+\gamma+\frac{\alpha_{0}^{0}}{C_{d}^{2}}\right)-\Gamma^{1 / 2}\right]\right\}^{1 / 2},
\end{aligned}
$$

$\bar{P}=P(1)$ and the \pm signs designate the waves propagating in the positive and negative directions, respectively. In the subsequent analysis we drop the use of double signs and choose the + sign that corresponds to waves leaving the boundary of the circular hole and propagating into the region $1<r<+\infty$. Equation (3.26) reveals that according to the Gurtin and Pipkin and the Chen and Gurtin theory, thermoelastic disturbances generate two wavefronts located at

$$
\begin{aligned}
& t=P_{1}(r)=\bar{P}+(r-1) P_{1}^{\prime}, \\
& t=P_{2}(r)=\bar{P}+(r-1) P_{2}^{\prime},
\end{aligned}
$$

propagating at constant speeds

$$
\begin{aligned}
& v_{1}=\frac{1}{P_{1}^{\prime}}=\left\{\frac{1}{2 \alpha_{0}^{0}}\left[\left(1+\gamma+\frac{\alpha_{0}}{C_{d}^{2}}\right)+\Gamma^{1 / 2}\right]\right\}^{-1 / 2}, \\
& v_{2}=\frac{1}{P_{2}^{\prime}}=\left\{\frac{1}{2 \alpha_{0}^{0}}\left[\left(1+\gamma+\frac{\alpha_{0}^{0}}{C_{d}^{2}}\right)-\Gamma^{1 / 2}\right]\right\}^{-1 / 2},
\end{aligned}
$$

$\left(v_{1}<v_{2}\right)$, respectively. In their study of one-dimensional progressive waves in thermoelastic half spaces Sawatzky and Moodie [14] have shown that the faster wavespeed is greater and the slower wavespeed is less than the speed of purely mechanical dilatational waves for the material. However, observing that $v_{1}$ is a decreasing and $v_{2}$ is an increasing function of the thermoelastic coupling constant, the inequalities

$$
\begin{aligned}
& v_{1}<\left\{\frac{1}{2 \alpha_{0}^{0}}\left[\left(1+\frac{\alpha_{0}^{0}}{C_{d}^{2}}\right)+\left|\frac{\alpha_{0}^{0}}{C_{d}^{2}}-1\right|\right]\right\}^{-1 / 2}, \\
& v_{2}>\left\{\frac{1}{2 \alpha_{0}^{0}}\left[\left(1+\frac{\alpha_{0}^{0}}{C_{d}^{2}}\right)-\left|\frac{\alpha_{0}^{0}}{C_{d}^{2}}-1\right|\right]\right\}^{-1 / 2} .
\end{aligned}
$$

obtained from (3.25) and (3.29) reveal that for $\alpha_{0}^{0} / C_{d}^{2}<1, v_{2}>C_{d}$, and $v_{1}<$ $\left(\alpha_{0}^{0}\right)^{1 / 2}$, where $\left(\alpha_{0}^{0}\right)^{1 / 2}$ is the speed of purely thermal waves for the material [17]. 
while for $\alpha_{0}^{0} / C_{d}^{2} \geq 1, v_{2}>\left(\alpha_{0}^{0}\right)^{1 / 2}$, and $v_{1}<C_{d}$. Therefore, the speed of the fast wave is not only greater than the speed of purely mechanical dilatational waves but also greater than the speed of purely thermal waves. Likewise, the speed of the slow wave is less than the smaller of the purely mechanical and purely thermal wavespeeds. Furthermore, setting $\gamma=0$ in (3.29) we find that for $\alpha_{0}^{0} / C_{d}^{2}<1, v_{2}=C_{d}$, and $v_{1}=\left(\alpha_{0}^{0}\right)^{1 / 2}$ whereas for $\alpha_{0}^{0} / C_{d}^{2} \geq 1, v_{2}=\left(\alpha_{0}^{0}\right)^{1 / 2}$, and $v_{1}=C_{d}$. These results lead us to the conclusion that for $\alpha_{0}^{0} / C_{d}^{2}<1$ the fast wave is a quasi-elastic wave and the slow wave is a quasi-thermal wave. For $\alpha_{0}^{0} / C_{d}^{2} \geq 1$ the roles of the fast and slow waves are reversed and the fast wave is a quasi-thermal wave while the slow wave is a quasi-elastic wave.

Returning to our original analysis we observe from the above results that the asymptotic wavefront expansions for $\Phi$ and $\theta$ should consist of the sum of expansions at each wavefront. Therefore, we replace the expansion (3.16) and (3.17) by

$$
\begin{aligned}
& \Phi(r, t)=\sum_{\ell=1}^{2} \sum_{n=2}^{\infty} \phi_{\ell n}(r) F_{n}\left(t-P_{\ell}(r)\right), \\
& \theta(r, t)=\sum_{\ell=1}^{2} \sum_{n=0}^{\infty} T_{\ell n}(r) F_{n}\left(t-P_{\ell}(r)\right),
\end{aligned}
$$

where $F_{n}$ satisfies (3.19) as before and the phase functions $P_{1}(r)$ and $P_{2}(r)$ are given by (3.28). When (3.32) is substituted into (3.5) the result is again (3.21) except that $P^{\prime}, T_{n}$ are replaced by $P_{1}^{\prime}, T_{1 n}$ and $P_{2}^{\prime}, T_{2 n}$. For $n=0,(3.21)$ gives the eikonal equation (3.23). Putting $n=1$ in (3.21) yields the first of the so-called transport equations which is

$$
T_{\ell_{0}}^{\prime}+\left\{\frac{1}{2 r}+\frac{\left(\left(P_{\ell}^{\prime}\right)^{2}-1 / C_{d}^{2}\right)}{2 P_{\ell}^{\prime}}\left[\frac{\alpha_{1}^{0}\left(P_{\ell}^{\prime}\right)^{2}-\beta_{0}^{0}}{\left(1+\gamma+\alpha_{0}^{0} / C_{d}^{2}\right)-2 \alpha_{0}^{0}\left(P_{\ell}^{\prime}\right)^{2}}\right]\right\} T_{\ell_{0}}=0 .
$$

Solving this equation we find

$$
T_{\ell_{0}}(r)=\bar{T}_{\ell_{0}} r^{-1 / 2} e^{-w_{\ell}(r-1)}
$$

where

$$
W_{\ell}=\frac{\left(\left(P_{\ell}^{\prime}\right)^{2}-1 / C_{d}^{2}\right)}{2 P_{\ell}^{\prime}}\left[\frac{\alpha_{1}^{0}\left(P_{\ell}^{\prime}\right)^{2}-\beta_{0}^{0}}{\left(1+\gamma+\alpha_{0}^{0} / C_{d}^{2}\right)-2\left(\alpha_{0}^{0}\left(P_{\ell}^{\prime}\right)^{2}\right.}\right], \quad \ell=1,2,
$$

and $\bar{T}_{\ell_{0}}=T_{\ell_{0}}(1)$. Thus, for a thermoelastic material whose thermomechanical behaviour is characterized by the constitutive equations of the linear theory of Gurtin and Pipkin and of Chen and Gurtin, a discontinuous change in temperature is attenuated at each wavefront according to (3.34) and (3.35). The identity

$$
\left(1+\gamma+\frac{\alpha_{0}^{0}}{C_{d}^{2}}\right)-2 \alpha_{0}^{0}\left(P_{l}^{\prime}\right)^{2}=(-1)^{\ell} \Gamma^{1 / 2},
$$


obtained from (3.27) and the previous discussion on the wavespeeds indicates that the sign of $W_{\ell}$ is completely determined by the sign of $\left(\alpha_{0}^{0}\left(P_{l}^{\prime}\right)^{2}-\beta_{0}^{0}\right)$. Therefore, in one-dimensional circular geometry, a discontinuous change in temperature decays with $r$ if

$$
\alpha_{1}^{0}\left(P_{\ell}^{\prime}\right)^{2}-\beta_{0}^{0} \leq 0
$$

and the restrictions (2.40) on $\alpha_{1}^{0}$ and $\beta_{0}^{0}$ guarantee that (3.37) always holds. As will be clear later, the condition (3.37) suffices also for discontinuous changes in $\varepsilon_{r r}, \sigma_{r r}$, and $\sigma_{\varphi \varphi}$ to decay to zero as $r$ increases.

The higher-order transport equations for $T_{\ell_{1}}, T_{\ell_{2}}, \ldots$, determined from (3.21) are now solved to yield

$$
\begin{array}{r}
T_{\ell n}(r)=r^{-1 / 2} e^{-W_{\ell}^{\prime}(r-1)}\left\{\bar{T}_{\ell n}+\int_{1}^{r}\left(r^{\prime}\right)^{1 / 2} e^{W_{\ell}^{\prime}\left(r^{\prime}-1\right)} Q_{\ell n}\left(r^{\prime}\right) d r^{\prime}\right\}, \\
\ell=1,2, j=1,2, \ldots,
\end{array}
$$

where

$$
Q_{\ell n}(r)=\frac{(-1)^{\ell}}{2 P_{\ell}^{\prime} \Gamma^{1 / 2}}\left\{\sum_{k=1}^{n}\left(L_{\ell k}^{1} T_{\ell, n-k}+L_{\ell k}^{2} T_{\ell, n-1-k}+L_{\ell k}^{3} T_{\ell, n-2-k}\right)\right\},
$$

and $L_{\ell k}^{1}, L_{\ell k}^{2}$, and $L_{\ell k}^{3}$ are the ordinary differential operators defined by

$$
\begin{aligned}
L_{\ell k}^{1} f= & {\left[(1+\gamma) \delta_{1 k}+\left(\frac{1}{C_{d}^{2}}-6\left(P_{\ell}^{\prime}\right)^{2}\right) \alpha_{k-1}^{0}\right]\left(f^{\prime \prime}+\frac{1}{r} f^{\prime}\right) } \\
& +P_{\ell}^{\prime}\left[\left(2\left(P_{\ell}^{\prime}\right)^{2}-\frac{1}{C_{d}^{2}}\right) \alpha_{k}^{0}-\beta_{k-1}^{0}\right]\left(2 f^{\prime}+\frac{1}{r} f\right) \\
& -\left(\left(P_{\ell}^{\prime}\right)^{2}-\frac{1}{C_{d}^{2}}\right)\left(\left(P_{\ell}^{\prime}\right)^{2} \alpha_{k+1}^{0}-\beta_{k}^{0}\right) f+\left(P_{\ell}^{\prime}\right)^{2} \alpha_{k-1}^{0} \frac{f}{r^{2}}, \\
L_{\ell k}^{2} f= & P_{\ell}^{\prime} \alpha_{k-1}^{0}\left(4 f^{\prime \prime \prime}+\frac{6}{r} f^{\prime \prime}-\frac{2}{r^{2}} f^{\prime}+\frac{1}{r^{3}} f\right)+\beta_{k-1}^{0}\left(f^{\prime \prime}+\frac{1}{r} f^{\prime}\right), \\
L_{\ell k}^{3} f= & -\alpha_{k-1}^{0}\left(f^{(i \prime \prime)}+\frac{2}{r} f^{\prime \prime \prime}-\frac{1}{r^{2}} f^{\prime \prime}+\frac{1}{r^{3}} f^{\prime}\right) .
\end{aligned}
$$

In (3.40), $\delta_{1 k}$ indicates the Kronecker delta. Since $T_{\ell n} \equiv 0$ for $n<0$, some of the summations in (3.39) have been extended to $k=n$. It can be proved by induction that the amplitude functions $T_{\ell n}$ are of the form

$$
T_{\ell n}(r)=r^{-1 / 2} e^{-n_{i}(r-1)} \sum_{j=-n}^{n} t_{\ell j n} r^{j}, \quad \ell=1,2, n=0,1,2, \ldots
$$


Substituting (3.41) into (3.38) and simplifying gives the recursion relation

$$
t_{\ell j n}=\left\{\begin{array}{c}
\frac{(-1)^{\ell}}{2 j P_{l}^{\prime} \Gamma^{1 / 2}} \sum_{k=1}^{n-|j|+1}\left(\sum_{m=0}^{2} A_{\ell k j}^{m} t_{\ell, j+1-m, n-k}+\sum_{m=0}^{3} B_{\ell k j}^{m} t_{\ell, j+2-m, n-1-k}\right. \\
\left.\quad+\sum_{m=0}^{4} C_{\ell k j}^{m} t_{\ell, j+3-m, n-2-k}\right), \quad j \neq 0, \quad-n \leq j \leq n, \\
\bar{T}_{\ell n}-\sum_{k=1}^{n}\left(t_{\ell,-k, n}+t_{\ell, k, n}\right), \quad j=0, n \geq 0, \\
0, \quad j<-n \text { or } j>n,
\end{array}\right.
$$

where

$$
\begin{aligned}
A_{\ell k j}^{0}= & \left(j+\frac{1}{2}\right)^{2}\left\{(1+\gamma) \delta_{1 k}+\left(\frac{1}{C_{d}^{2}}-6\left(P_{\ell}^{\prime}\right)^{2}\right) \alpha_{k-1}^{0}\right\}+\left(P_{\ell}^{\prime}\right)^{2} \alpha_{k-1}^{0}, \\
A_{\ell k j}^{1}= & -2 j\left\{W_{\ell}\left[(1+\gamma) \delta_{1 k}+\left(\frac{1}{C_{d}^{2}}-6\left(P_{\ell}^{\prime}\right)^{2}\right) \alpha_{k-1}^{0}\right]\right. \\
& \left.-P_{\ell}^{\prime}\left[\left(2\left(P_{\ell}^{\prime}\right)^{2}-\frac{1}{C_{d}^{2}}\right) \alpha_{k}^{0}-\beta_{k-1}^{0}\right]\right\}, \\
A_{\ell k j}^{2}= & W_{\ell}^{2}\left\{(1+\gamma) \delta_{1 k}+\left(\frac{1}{C_{d}^{2}}-6\left(P_{\ell}^{\prime}\right)^{2}\right) \alpha_{k-1}^{0}\right\} \\
& -2 W_{\ell} P_{\ell}^{\prime}\left\{\left(2\left(P_{\ell}^{\prime}\right)^{2}-\frac{1}{C_{d}^{2}}\right) \alpha_{k}^{0}-\beta_{k-1}^{0}\right\} \\
& -\left(\left(P_{\ell}^{\prime}\right)^{2}-\frac{1}{C_{d}^{2}}\right)\left(\alpha_{k+1}^{0}\left(P_{\ell}^{\prime}\right)^{2}-\beta_{k}^{0}\right), \\
B_{\ell k j}^{0}= & P_{\ell}^{\prime} \alpha_{k-1}^{0} M_{4}(j+2), \\
B_{\ell k j}^{1}= & \beta_{k-1}^{0} M_{2}(j+1)-2 P_{\ell}^{\prime} W_{\ell} \alpha_{k-1}^{0} M_{3}(j+1), \\
B_{\ell k j}^{2}= & \left\{6 P_{\ell}^{\prime} W_{\ell}^{2} \alpha_{k-1}^{0}-W_{\ell} \beta_{k-1}^{0}\right\} M_{1}(j), \\
B_{\ell k j}^{3}= & W_{\ell}^{2} \beta_{k-1}^{0}-4 P_{\ell}^{\prime} W_{\ell}^{3} \alpha_{k-1}^{0}, \\
C_{\ell k j}^{0}= & -\alpha_{k-1}^{0} M_{5}(j+3), \quad C_{\ell k j}^{1}=W_{\ell} \alpha_{k-1}^{0} M_{4}(j+2), \\
C_{\ell k j}^{2}= & -W_{\ell}^{2} \alpha_{k-1}^{0} M_{3}(j+1), \quad C_{\ell k j}^{2}=2 W_{\ell}^{3} \alpha_{k-1}^{0} M_{1}(j), \\
C_{\ell k j}^{4}= & -W_{\ell}^{4} \alpha_{k-1}^{0} .
\end{aligned}
$$

In the above equations the auxiliary functions $M_{1}(j)$ to $M_{5}(j)$ are

$$
\begin{aligned}
& M_{1}(j)=2 K_{1}(j)+1, \\
& M_{2}(j)=K_{1}(j)+K_{2}(j), \\
& M_{3}(j)=6 K_{2}(j)+6 K_{1}(j)-1, \\
& M_{4}(j)=4 K_{3}(j)+6 K_{2}(j)-2 K_{1}(j)+1, \\
& M_{5}(j)=K_{4}(j)+2 K_{3}(j)-K_{2}(j)+K_{1}(j),
\end{aligned}
$$


where

$$
\begin{gathered}
K_{1}(j)=j-\frac{1}{2}, \quad K_{2}(j)=\left(j-\frac{1}{2}\right)\left(j-\frac{3}{2}\right), \\
K_{3}(j)=\left(j-\frac{1}{2}\right)\left(j-\frac{3}{2}\right)\left(j-\frac{5}{2}\right), \\
K_{4}(j)=\left(j-\frac{1}{2}\right)\left(j-\frac{3}{2}\right)\left(j-\frac{5}{2}\right)\left(j-\frac{7}{2}\right) .
\end{gathered}
$$

Repeating the above procedure for the amplitude coefficients $\phi_{\ell n}(r)$ in the expansion (3.31) for $\Phi(r, t)$, we then obtain

$$
\phi_{\ell n}(r)=r^{-1 / 2} e^{-w_{\ell}(r-1)} \sum_{j=-(n-2)}^{n-2} \varphi_{\ell j n} r^{j}, \quad \ell=1,2, n=2,3, \ldots,
$$

where

$$
\varphi_{\ell j n}=\left\{\begin{array}{l}
\frac{(-1)^{\prime}}{2 j P_{\ell}^{\prime} \Gamma^{1 / 2}} \sum_{k=1}^{n-|j|-1}\left(\sum_{m=0}^{2} A_{\ell k j}^{m} \varphi_{\ell, j+1-m, n-k}+\sum_{m=0}^{3} B_{\ell k j}^{m} \varphi_{\ell, j+2-m, n-1-k}\right. \\
\left.\quad+\sum_{m=0}^{4} C_{\ell k j}^{m} \varphi_{\ell, j+3-m, n-2-k}\right), \quad j \neq 0, \quad-(n-2) \leq j \leq n-2, \\
\bar{\phi}_{\ell n}-\sum_{k=1}^{n-2}\left(\varphi_{\ell,-k, n}+\varphi_{\ell, k, n}\right), \quad j=0, \quad n \geq 2, \\
0, \quad j<-(n-2) \quad \text { or } j>(n-2) .
\end{array}\right.
$$

However, the amplitude coefficients $T_{\ell n}(r)$ and $\phi_{\ell n}(r)$ are not free but connected through the field equations (3.2) and (3.3) for $\theta$ and $\Phi$. It is easy to verify that inserting the expansions (3.31) and (3.32) into one of the field equations, say (3.2), after some algebra the relationship between $T_{\ell n}$ and $\Phi_{\ell_{n}}$ can be found as

$$
\begin{aligned}
& \left(j+\frac{3}{2}\right)^{2} \varphi_{\ell, j+2, n}-2(j+1) W_{\ell} \varphi_{\ell, j+1, n}+W_{\ell}^{2} \varphi_{\ell, j, n} \\
& \quad-2(j+1) P_{\ell}^{\prime} \varphi_{\ell, j+1, n+1}+2 P_{\ell}^{\prime} W_{\ell} \varphi_{\ell, j, n+1}+\left(\left(P_{\ell}^{\prime}\right)^{2}-\frac{1}{C_{d}^{2}}\right) \varphi_{\ell, j, n+2}=t_{\ell, n n}, \\
& \ell=1,2, j=0, \pm 1, \pm 2, \ldots, \pm n, n=0,1,2, \ldots
\end{aligned}
$$

For $j=0$, the use of (3.42) and (3.47) in (3.48) gives the relationship between $\bar{T}_{\ell n}$ and $\bar{\phi}_{\ell n}$ which is

$$
\begin{aligned}
\left(\left(P_{\ell}^{\prime}\right)^{2}\right. & \left.-\frac{1}{C_{d}^{2}}\right) \bar{\phi}_{\ell, n+2}+2 P_{\ell}^{\prime} W_{\ell} \bar{\phi}_{\ell, n+1}+w_{\ell}^{2} \bar{\phi}_{\ell, n}-\bar{T}_{\ell, n} \\
= & \left(\left(P_{\ell}^{\prime}\right)^{2}-\frac{1}{C_{d}^{2}}\right) \sum_{k=1}^{n}\left(\varphi_{\ell,-k, n+2}+\varphi_{\ell, k, n+2}\right)+2 P_{\ell}^{\prime} W_{\ell} \sum_{k=1}^{n-1}\left(\varphi_{\ell,-k, n+1}+\varphi_{\ell, k, n+1}\right) \\
& +W_{\ell}^{2} \sum_{k=1}^{n-2}\left(\varphi_{\ell,-k, n}+\varphi_{\ell, k, n}\right)-\frac{9}{4} \varphi_{\ell, 2, n}+2 W_{\ell} \varphi_{\ell, 1, n}+2 P_{\ell}^{\prime} \varphi_{\ell, 1, n+1} \\
& -\sum_{k=1}^{n}\left(t_{\ell,-k, n}+t_{\ell, k, n}\right), \quad \ell=1,2, n=0,1,2, \ldots
\end{aligned}
$$


On the other hand, the recursive use of (3.42) and (3.47), together with (3.49), reduces (3.48) to an identity for $j= \pm 1, \pm 2, \ldots, \pm n$.

So far, we have obtained the solution of the thermoelastic problem (3.5) subject to initial and boundary conditions (3.10) and (3.11). This solution is given by (3.31) and (3.32) where $P_{\ell}(r), T_{\ell n}(r)$, and $\phi_{\ell n}(r)$ are determined from (3.27), (3.28), and (3.41)-(3.47). The initial values $\bar{P}, \bar{T}_{\ell n}$, and $\bar{\phi}_{\ell n}$ are to be found from the source conditions (3.10) and (3.11) with the use of (3.49). In order to complete the solution, the waveform $F_{0}$, which in turn fixes the wavefunctions $F_{n}$ from the relations (3.19), should be determined from the source conditions as well.

Let us consider the following source conditions

$$
\begin{aligned}
& \theta(1, t)=H(t), \quad \frac{\partial^{2} \Phi(r, t)}{\partial r^{2}}+\left.\frac{\nu}{r} \frac{\partial \Phi(r, t)}{\partial r}\right|_{r=1}=0, \\
& \theta(1, t)=0, \quad \frac{\partial^{2} \Phi(r, t)}{\partial r^{2}}+\left.\frac{\nu}{r} \frac{\partial \Phi(r, t)}{\partial r}\right|_{r=1}=H(t),
\end{aligned}
$$

which correspond to purely thermal and purely mechanical unit step disturbances, respectively. We shall refer to these canonical problems as Problem 1 and Problem 2 for convenience. The solution to the problem corresponding to the general source conditions (3.10), (3.11) can then be obtained with the appropriate superposition of the solutions to Problems 1 and 2 and the use of Duhamel's theorem.

Let $\theta^{(1)}, \Phi^{(1)}$ and $\theta^{(2)}, \Phi^{(2)}$ be the respective solutions to the canonical problems. Substituting (3.31) and (3.32) into (3.50) and (3.51) and using (3.42), (3.47) we get

$$
\begin{aligned}
\sum_{\ell=1}^{2} \sum_{n=0}^{\infty} \bar{T}_{\ell n}^{(m)} F_{n}^{(m)}\left(t-\bar{P}_{\ell}^{(m)}\right)= \begin{cases}H(t), & m=1, \\
0, & m=2,\end{cases} \\
\sum_{\ell=1}^{2} \sum_{n=0}^{\infty} \sum_{j=-n}^{n}\left\{\left(P_{\ell}^{\prime}\right)^{2} \varphi_{\ell, j, n+2}^{(m)}+2 P_{\ell}^{\prime} W_{\ell} \varphi_{\ell, j, n+1}^{(m)}+W_{\ell}^{2} \varphi_{\ell j n}^{(m)}\right. \\
+\left[\left(j+\frac{3}{2}\right)^{2}-2\left(j+\frac{3}{2}\right) \frac{C_{s}^{2}}{C_{d}^{2}}\right] \varphi_{\ell, j+2, n}^{(m)} \\
-\left[2(j+1)-2 \frac{C_{s}^{s}}{C_{d}^{2}}\right] W_{\ell} \varphi_{\ell, j+1, n}^{(m)} \\
\left.-\left[2(j+1)-2 \frac{C_{s}^{2}}{C_{d}^{2}}\right] P_{\ell}^{\prime} \varphi_{\ell, j+1, n+1}\right\} F_{n}^{(m)}\left(t-\bar{P}_{\ell}^{(m)}\right) \\
= \begin{cases}0, & m=1, \\
H(t), & m=2 .\end{cases}
\end{aligned}
$$


From these two equations we then choose

$$
\begin{gathered}
\bar{P}_{\ell}^{(m)}=0, \quad F_{0}^{(m)}(t)=H(t), \quad m=1,2, \ell=1,2 \\
\bar{T}_{10}^{(1)}+\bar{T}_{20}^{(1)}=1, \quad\left(P_{1}^{\prime}\right)^{2} \bar{\phi}_{12}^{(1)}+\left(P_{2}^{\prime}\right)^{2} \phi_{12}^{(12)}=0, \\
\bar{T}_{10}^{(2)}+\bar{T}_{20}^{(2)}=0, \quad\left(P_{1}^{\prime}\right)^{2} \bar{\phi}_{12}^{(2)}+\left(P_{2}^{\prime}\right)^{2} \phi_{22}^{(2)}=1, \\
\sum_{\ell=1}^{2} \bar{T}_{\ell n}^{(m)}=0, \quad m=1,2, n=1,2, \ldots, \\
\sum_{\ell=1}^{2} \sum_{j=-n}^{n}\left\{\left(P_{\ell}^{\prime}\right)^{2} \varphi_{\ell, j, n+1}^{(m)}+2 P_{\ell}^{\prime} E_{\ell} \varphi_{\ell, j, n+1}^{(m)}+W_{\ell}^{2} \varphi_{\ell j n}^{(m)}\right. \\
+\left[\left(j+\frac{3}{2}\right)^{2}-2\left(j+\frac{3}{2}\right) \frac{C_{s}^{2}}{C_{d}^{2}}\right] \varphi_{\ell, j+2, n}^{(m)} \\
-\left[2(j+1)-2 \frac{C_{s}^{2}}{C_{d}^{2}}\right] W_{\ell} \varphi_{\ell, j+1, n}^{(m)} \\
\left.-\left[2(j+1)-2 \frac{C_{s}^{2}}{C_{d}^{2}}\right] P_{\ell}^{\prime} \varphi_{\ell, j+1, n+1}\right\}=0, \quad m=1,2, n=1,2, \ldots
\end{gathered}
$$

We further note that for more general source conditions where the Heaviside step function $H(t)$ is replaced by arbitrary functions $f^{(1)}(t)$ and $f^{(2)}(t)$ in Problems 1 and 2 , respectively, the wavefunctions $F_{n}^{(m)}(t), n \geq 1$ are determined from the waveform $F_{0}^{(m)}(t)=f^{(m)}(t)$ with the use of Duhamel's theorem [14] as

$$
F_{n}^{(m)}(t)=\frac{H(t)}{n !} \frac{\partial}{\partial t} \int_{0}^{t}(t-\xi)^{n} f^{(m)}(\xi) d \xi .
$$

It is easy to show from (3.49), (3.57), and (3.58) that the coefficients $\bar{\phi}_{\ell, n+2}^{(m)}$ and $\bar{T}_{\ell, n}^{(m)}$ for $n=1,2, \ldots$, are

$$
\begin{aligned}
\bar{\phi}_{\ell, n+2}^{(m)}= & \sum_{k=1}^{n}\left(\varphi_{\ell,-k, n+2}^{(m)}+\varphi_{\ell, k, n+2}^{(m)}\right)+(-1)^{\ell} \alpha_{0}^{0} \Gamma^{-1 / 2} \\
& \times \sum_{q=1}^{2}\left\{2 P_{q}^{\prime} W_{q} \varphi_{q, 0, n+1}^{(m)}+W_{q}^{2} \varphi_{q, 0, n}^{(m)}+\left(\frac{9}{4}-3 C_{s}^{2}\left(\frac{1}{C_{d}^{2}}-\left(P_{3-\ell}^{\prime}\right)^{2}\right)\right) \varphi_{q, 2, n}^{(m)}\right. \\
& -2\left(1-C_{s}^{2}\left(\frac{1}{C_{d}^{2}}-\left(P_{3-\ell}^{\prime}\right)^{2}\right)\right) W_{q} \varphi_{q, 1, n}^{(m)} \\
& -2\left(1-C_{s}^{2}\left(\frac{1}{C_{d}^{2}}-\left(P_{3-\ell}^{\prime}\right)^{2}\right)\right) P_{q}^{\prime} \varphi_{q, 1, n+1}^{(m)} \\
& \left.+C_{d}^{2}\left(\frac{1}{C_{d}^{2}}-\left(P_{3-\ell}^{\prime}\right)^{2}\right) S_{q n}^{(m)}+C_{d}^{2}\left(P_{3-\ell}^{\prime}\right)^{2} \sum_{k=1}^{n}\left(t_{q,-k, n}+t_{q, k, n}\right)\right\}
\end{aligned}
$$




$$
\begin{gathered}
\bar{T}_{\ell n}^{(m)}=\sum_{k=1}^{n}\left(t_{\ell,-k, n}^{(m)}+t_{\ell, k, n}^{(m)}\right)+\left(\left(P_{\ell}^{\prime}\right)^{2}-\frac{1}{C_{d}^{2}}\right) \varphi_{\ell, 0, n+2}^{(m)}+2 P_{\ell}^{\prime} W_{\ell} \varphi_{\ell, 0, n+1}^{(m)} \\
+W_{\ell}^{2} \varphi_{\ell, 0, n}^{(m)}+\frac{9}{4} \varphi_{\ell, 2, n}^{(m)}-2 W_{\ell} \varphi_{\ell, 1, n}^{(m)}-2 P_{\ell}^{\prime} \varphi_{\ell, 1, n}^{(m)}-2 P_{\ell}^{\prime} \varphi_{\ell, 1, n+1}^{(m)}, \\
m=1,2, \quad \ell=1,2, n=1,2, \ldots,
\end{gathered}
$$

where

$$
\begin{aligned}
S_{\ell n}^{(m)}=\sum_{\substack{j=-n \\
j \neq 0}}^{n} & \left\{\left(P_{\ell}^{\prime}\right)^{2} \varphi_{\ell, j, n+2}^{(m)}+2 P_{\ell}^{\prime} W_{\ell} \varphi_{\ell, j, n+1}^{(m)}+W_{\ell}^{2} \varphi_{\ell j n}^{(m)}\right. \\
& +\left[\left(j+\frac{3}{2}\right)^{2}-2\left(j+\frac{3}{2}\right) \frac{C_{s}^{2}}{C_{d}^{2}}\right] \varphi_{\ell, j+2, n}^{(m)}-\left[2(j+1)-2 \frac{C_{s}^{2}}{C_{d}^{2}}\right] W_{\ell} \varphi_{\ell, j+1, n}^{(m)} \\
& \left.-\left[2(j+1)-2 \frac{C_{s}^{2}}{C_{d}^{2}}\right] P_{\ell}^{\prime} \varphi_{\ell, j+1, n+1}\right\}, \quad m=1,2, n=1,2, \ldots
\end{aligned}
$$

For $n=0$, the initial values $\bar{T}_{\ell_{0}}^{(m)}, \bar{\phi}_{\ell_{2}}^{(m)}$ also obtained from the same equations are

$$
\begin{array}{ccc}
\bar{T}_{10}^{(1)}=\frac{C_{d}^{2}\left(P_{2}^{\prime}\right)^{2}\left(\left(P_{1}^{\prime}\right)^{2}-1 / C_{d}^{2}\right)}{\left(P_{1}^{\prime}\right)^{2}-\left(P_{2}^{\prime}\right)^{2}}, & \bar{T}_{20}^{(1)}=\frac{C_{d}^{2}\left(P_{1}^{\prime}\right)^{2}\left(1 / C_{d}^{2}-\left(P_{2}^{\prime}\right)^{2}\right)}{\left(P_{1}^{\prime}\right)^{2}-\left(P_{2}\right)^{2}}, \\
\bar{\phi}_{12}^{(1)}=\frac{C_{d}^{2}\left(P_{2}^{\prime}\right)^{2}}{\left(P_{1}^{\prime}\right)^{2}-\left(P_{2}^{\prime}\right)^{2}}, & \bar{\phi}_{22}^{(1)}=-\frac{C_{d}^{2}\left(P_{1}^{\prime}\right)^{2}}{\left(P_{1}^{\prime}\right)^{2}-\left(P_{2}^{\prime}\right)^{2}},
\end{array}
$$

and

$$
\begin{gathered}
\bar{T}_{10}^{(2)}=\bar{T}_{20}^{(2)}=\frac{C_{d}^{2}\left(\left(P_{1}^{\prime}\right)^{2}-1 / C_{d}^{2}\right)\left(1 / C_{d}^{2}-\left(P_{2}^{\prime}\right)^{2}\right)}{\left(P_{1}^{\prime}\right)^{2}-\left(P_{2}^{\prime}\right)^{2}}, \\
\bar{\phi}_{12}^{(2)}=\frac{C_{d}^{2}\left(1 / C_{d}^{2}-\left(P_{2}^{\prime}\right)^{2}\right)}{\left(P_{1}^{\prime}\right)^{2}-\left(P_{2}^{\prime}\right)^{2}}, \quad \bar{\phi}_{22}^{(2)}=\frac{C_{d}^{2}\left(\left(P_{1}^{\prime}\right)^{2}-1 / C_{d}^{2}\right)}{\left(P_{1}^{\prime}\right)^{2}-\left(P_{2}^{\prime}\right)^{2}} .
\end{gathered}
$$

Thus the solutions of the canonical problems (3.50), (3.51) are given by

$$
\begin{gathered}
\theta^{(m)}(r, t)=\sum_{\ell=1}^{2} r^{-1 / 2} e^{-w_{\ell}(r-1)}\left\{\sum_{n=0}^{\infty} \frac{1}{n !}\left(t-(r-1) P_{\ell}^{\prime}\right)^{n} \sum_{j=-n}^{n} t_{\ell j n}^{(m)} r^{j}\right\} H\left(t-(r-1) P_{\ell}^{\prime}\right), \\
m=1,2, \quad(3.64) \\
\Phi^{(m)}(r, t)=\sum_{\ell=1}^{2} r^{-1 / 2} e^{-w_{\ell}(r-1)}\left\{\sum_{n=2}^{\infty} \frac{1}{n !}\left(t-(r-1) P_{\ell}^{\prime}\right)^{n} \sum_{j=-n}^{n} \varphi_{\ell j n}^{(m)} r^{j}\right\} H\left(t-(r-1) P_{\ell}^{\prime}\right), \\
m=1,2, \quad 3.65)
\end{gathered}
$$

where the wavefunctions $F_{n}$ are obtained from (3.54) and (3.59) with $f^{(m)}=1$, $t_{\ell j n}^{(m)}, \varphi_{\ell j n}^{(m)}$ from (3.42), (3.47) augmented by (3.60) for $\bar{T}_{\ell n}^{(m)}, \bar{\phi}_{\ell n}^{(m)}$. The strains $\varepsilon_{r r}^{(m)}, \varepsilon_{\varphi \varphi}^{(m)}$ can now be determined from the relevant strain-displacement relations 
whereas the stresses $\sigma_{r r}^{(m)}, \sigma_{\varphi \varphi}^{(m)}$ can be found from (3.7), (3.8) together with (3.64) and (3.65).

We now consider the following source condition for shear waves:

$$
\left.\frac{C_{s}^{2}}{C_{d}^{2}}\left(\frac{\partial u_{\varphi}}{\partial r}-\frac{u_{\varphi}}{r}\right)\right|_{r=1}=H(t)
$$

which we shall label as Problem 3. Proceeding as for the thermoelastic waves we find that the rays associated with shear waves are

$$
S(r)=\bar{S} \pm \frac{r-1}{C_{S}}, \quad \bar{S}=S(1),
$$

where the \pm signs are associated with outgoing and incoming waves, respectively. We again choose the + sign which corresponds to the waves leaving the boundary of the circular hole in the positive radial direction. The amplitude functions in (3.18) for $u_{\varphi}(r, t)$ are, on the other hand,

$$
U_{n}(r)=r^{-1 / 2} \sum_{j=0}^{n-1} u_{j n} r^{-j}, \quad n \geq 1
$$

where

$$
u_{j n}= \begin{cases}K(j) u_{j-1, n-1}, & \text { if } 1 \leq j \leq n-1, \\ -\frac{C_{d}^{2}}{C_{s}}, & \text { if } j=0, n=1, \\ -\sum_{j=1}^{n}\left[1+C_{s}(j-1 / 2)+K(j)\right] u_{j-1, n-1}, & \text { if } j=0, n \geq 2 \\ 0, & \text { if } j<0 \text { or } j>n-1\end{cases}
$$

and

$$
K(j)=\left(C_{s} / 2 j\right)\left[1-(j-1 / 2)^{2}\right]
$$

We have chosen

$$
\bar{S}=0, \quad F_{0}(t)=H(t)
$$

from the source condition (3.66). We observe that if $F_{0}(t)=f(t)$, for arbitrary $f(t)$, the wavefunctions $F_{n}(t)$ are again

$$
F_{n}(t)=\frac{H(t)}{n !} \frac{\partial}{\partial t} \int_{0}^{\infty}(t-\xi)^{n} f(\xi) d \xi .
$$

The complete expansion of the solution to Problem 3 is then given by

$$
u_{\varphi}(r, t)=\left\{r^{-1 / 2} \sum_{n=1}^{\infty} \frac{1}{n !}\left(t-\frac{r-1}{C_{s}}\right) \sum_{j=0}^{n-1} u_{j n} r^{-j}\right\} H\left(t-\frac{r-1}{C_{s}}\right),
$$

where $F_{n}$ are obtained from (3.72) with $f=1$. The strain $\varepsilon_{r \varphi}$ and the stress $\sigma_{r \varphi}$ corresponding to the displacement (3.73) may be composed as before.

In order to study the propagation of discontinuities in temperature, strains, and stresses generated by the source conditions (3.50), (3.51), and (3.66), we now introduce the usual bracket notation

$$
[f]_{t=A(r)}=\left.f(r, t)\right|_{t=A^{+}(r)}-\left.f(r, t)\right|_{t=A^{-}(r)},
$$


which represents the discontinuity of a function $f(r, t)$ across a wavefront $t=A(r)$. We first recall that the disturbance of Problem 3 generates shear waves only. The discontinuities in $\varepsilon_{r \varphi}$ and $\sigma_{r \varphi}$ introduced by (3.66) evolve into discontinuities in these variables across the wavefront $t=(r-1) / C_{s}$. These discontinuities are

$$
\left[\varepsilon_{r \varphi}\right]_{t=(r-1) / C_{s}}=\frac{C_{d}^{2}}{C_{s}^{2}} r^{-1 / 2}, \quad\left[\sigma_{r \varphi}\right]_{t=(r-1) / C_{s}}=r^{-1 / 2} .
$$

The disturbances (3.50) and (3.51), on the other hand, generate thermoelastic waves with the two wavefronts $t=(r-1) P_{1}^{\prime}$ and $t=(r-1) P_{2}^{\prime}$. In particular, we find that $\varepsilon_{\varphi \varphi}$ is continuous whereas $\theta, \varepsilon_{r r}, \sigma_{r r}$, and $\sigma_{\varphi \varphi}$ exhibit finite jump discontinuities across each wavefront. For Problem 1 where the disturbance is purely thermal

$$
\begin{aligned}
& {\left[\theta^{(1)}\right]_{t=(r-1) P_{1}^{\prime}}=\frac{C_{d}^{2}\left(P_{2}^{\prime}\right)\left(\left(P_{1}^{\prime}\right)^{2}-1 / C_{d}^{2}\right)}{\left(P_{1}^{\prime}\right)^{2}-\left(P_{2}^{\prime}\right)^{2}} r^{-1 / 2} e^{-W_{1}(r-1)},} \\
& {\left[\varepsilon_{r r}^{(1)}\right]_{t=(r-1) P_{1}^{\prime}}=\frac{C_{d}^{2}\left(P_{1}^{\prime}\right)^{2}\left(P_{2}^{\prime}\right)^{2}}{\left(P_{1}^{\prime}\right)^{2}-\left(P_{2}^{\prime}\right)^{2}} r^{-1 / 2} e^{-W_{1}(r-1)},} \\
& {\left[\sigma_{r r}^{(1)}\right]_{t=(r-1) P_{1}^{\prime}}=\frac{\left(P_{2}^{\prime}\right)^{2}}{\left(P_{1}^{\prime}\right)^{2}-\left(P_{2}^{\prime}\right)^{2}} r^{-1 / 2} e^{-W_{1}(r-1)},} \\
& {\left[\sigma_{\varphi \varphi}^{(1)}\right]_{t=(r-1) P_{1}^{\prime}}=\frac{2 C_{s}^{2}\left(P_{2}^{\prime}\right)^{2}\left(1 / 2 C_{s}^{2}-\left(P_{1}^{\prime}\right)^{2}\right)}{\left(P_{1}^{\prime}\right)^{2}-\left(P_{2}^{\prime}\right)^{2}} r^{-1 / 2} e^{-W_{1}(r-1)},}
\end{aligned}
$$

and

$$
\begin{aligned}
& {\left[\theta^{(1)}\right]_{t=(r-1) P_{2}^{\prime}}=\frac{C_{d}^{2}\left(P_{1}^{\prime}\right)^{2}\left(1 / C_{d}^{2}-\left(P_{2}^{\prime}\right)^{2}\right)}{\left(P_{1}^{\prime}\right)^{2}-\left(P_{2}^{\prime}\right)^{2}} r^{-1 / 2} e^{-W_{2}(r-1)},} \\
& {\left[\varepsilon_{r r}^{(1)}\right]_{t=(r-1) P_{2}^{\prime}}=-\frac{C_{d}^{2}\left(P_{1}^{\prime}\right)^{2}\left(P_{2}^{\prime}\right)^{2}}{\left(P_{1}^{\prime}\right)^{2}-\left(P_{2}^{\prime}\right)^{2}} r^{-1 / 2} e^{-W_{2}(r-1)},} \\
& {\left[\sigma_{r r}^{(1)}\right]_{t=(r-1) P_{2}^{\prime}}=-\frac{\left(P_{1}^{\prime}\right)^{2}}{\left(P_{1}^{\prime}\right)^{2}-\left(P_{2}^{\prime}\right)^{2}} r^{-1 / 2} e^{-W_{2}(r-1)},} \\
& {\left[\sigma_{\varphi \varphi}^{(1)}\right]_{t=(r-1) P_{2}^{\prime}}=\frac{2 C_{s}^{2}\left(P_{1}^{\prime}\right)^{2}\left(\left(P_{2}^{\prime}\right)^{2}-1 / 2 C_{s}^{2}\right)}{\left(P_{1}^{\prime}\right)^{2}-\left(P_{2}^{\prime}\right)^{2}} r^{-1 / 2} e^{-W_{s}(r-1)} .}
\end{aligned}
$$

For Problem 2, that is, for a purely mechanical unit step disturbance

$$
\begin{aligned}
& {\left[\theta^{(2)}\right]_{t=(r-1) P_{1}^{\prime}}=\frac{C_{d}^{2}\left(\left(P_{1}^{\prime}\right)^{2}-1 / C_{d}^{2}\right)\left(1 / C_{d}^{2}-\left(P_{2}^{\prime}\right)^{2}\right)}{\left(P_{1}^{\prime}\right)^{2}-\left(P_{2}^{\prime}\right)^{2}} r^{-1 / 2} e^{-W_{1}(r-1)},} \\
& {\left[\varepsilon_{r r}^{(2)}\right]_{t=(r-1) P_{1}^{\prime}}=\frac{C_{d}^{2}\left(P_{1}^{\prime}\right)^{2}\left(1 / C_{d}^{2}-\left(P_{2}^{\prime}\right)^{2}\right)}{\left(P_{1}^{\prime}\right)^{2}-\left(P_{2}^{\prime}\right)^{2}} r^{-1 / 2} e^{-W_{1}(r-1)},} \\
& {\left[\sigma_{r r}^{(2)}\right]_{t=(r-1) P_{1}^{\prime}}=\frac{\left(1 / C_{d}^{2}-\left(P_{2}^{\prime}\right)^{2}\right)}{\left(P_{1}^{\prime}\right)^{2}-\left(P_{2}^{\prime}\right)^{2}} r^{-1 / 2} e^{-W_{1}(r-1)},} \\
& {\left[\sigma_{\varphi \varphi}^{(2)}\right]_{t=(r-1) P_{1}^{\prime}}=\frac{2 C_{s}^{2}\left(1 / 2 C_{s}^{2}-\left(P_{1}^{\prime}\right)^{2}\right)\left(1 / C_{d}^{2}-\left(P_{2}^{\prime}\right)^{2}\right)}{\left(P_{1}^{\prime}\right)^{2}-\left(P_{2}^{\prime}\right)^{2}} r^{-1 / 2} e^{-W_{1}(r-1)},}
\end{aligned}
$$


and

$$
\begin{aligned}
& {\left[\theta^{(2)}\right]_{t=(r-1) P_{2}^{\prime}}=-\frac{C_{d}^{2}\left(\left(P_{1}^{\prime}\right)^{2}-1 / C_{d}^{2}\right)\left(1 / C_{d}^{2}-\left(P_{2}^{\prime}\right)^{2}\right)}{\left(P_{1}^{\prime}\right)^{2}-\left(P_{2}^{\prime}\right)^{2}} r^{-1 / 2} e^{-W_{2}(r-1)},} \\
& {\left[\varepsilon_{r r}^{(2)}\right]_{t=(r-1) P_{2}^{\prime}}=\frac{C_{d}^{2}\left(P_{2}^{\prime}\right)^{2}\left(\left(P_{1}^{\prime}\right)^{2}-1 / C_{d}^{2}\right)}{\left(P_{1}^{\prime}\right)^{2}-\left(P_{2}^{\prime}\right)^{2}} r^{-1 / 2} e^{-W_{2}^{\prime}(r-1)},} \\
& {\left[\sigma_{r r}^{(2)}\right]_{t=(r-1) P_{2}^{\prime}}=\frac{\left(\left(P_{1}^{\prime}\right)^{2}-1 / C_{d}^{2}\right)}{\left(P_{1}^{\prime}\right)^{2}-\left(P_{2}^{\prime}\right)^{2} r^{-1 / 2} e^{w_{2}(r-1)},}} \\
& {\left[\sigma_{\varphi \varphi}^{(2)}\right]_{t=(r-1) P_{2}^{\prime}}=\frac{2 C_{s}^{2}\left(1 / 2 C_{s}^{2}-\left(P_{2}^{\prime}\right)^{2}\right)\left(\left(P_{1}^{\prime}\right)^{2}-1 / C_{d}^{2}\right)}{\left(P_{1}^{\prime}\right)^{2}\left(P_{2}^{\prime}\right)^{2}} r^{-1 / 2} e^{-W_{2}(r-1)}}
\end{aligned}
$$

Remarking that $\left(P_{1}^{\prime}\right)^{2}>1 / C_{d}^{2}$ and $1 / 2 C_{s}^{2}>1 / C_{d}^{2}>\left(P_{2}^{\prime}\right)^{2}$ we see from (3.76), (3.77), (3.78), and (3.79) that for both canonical problems the jumps in $\theta, \varepsilon_{r r}$, and $\sigma_{r r}$ are all of the same sign at the slower wavefront, whereas at the faster wavefront, the jump for $\theta$ is of opposite sign to those for $\varepsilon_{r r}, \sigma_{r r}$, and $\sigma_{\varphi \varphi}$. For both canonical problems the sign of $\left[\sigma_{\varphi \varphi}\right]_{t=(r-1) P_{1}^{\prime}}$ depends on the relative magnitudes of $\left(P_{1}^{\prime}\right)^{2}$ and $1 / 2 C_{s}^{2}$. Therefore, for $\left(P_{1}^{\prime}\right)^{2}<1 / 2 C_{s}^{2}$, that is, for $v_{1}^{2}>2 C_{s}^{2}$ the jumps in $\theta$ and $\sigma_{\varphi \varphi}$ are of the same sign whereas for $\left(P_{1}^{\prime}\right)^{2}>1 / 2 C_{s}^{2}$ or, equivalently, $v_{1}^{2}<2 C_{s}^{2}$ they are of opposite signs at the slower wavefront. These results provide further verification to the related results of Sawatzky and Moodie [14] as well as offering new information pertinent to one-dimensional circular geometry.

The ray-series method employed in this paper is suitable not only for the study of the propagation characteristics of thermoelastic disturbances but also for numerical evaluation of the solution behind the wavefronts. In the next section we present numerical results for the case in which the present theory reduces to the linear theory of Lord and Shulman [7].

4. Numerical results. In this section we specify the relaxation functions of the thermoelastic plate as

$$
\alpha(t)=\frac{e^{-t / \tau}}{\tau}, \quad \beta(t)=0 .
$$

The relaxation functions given by $(4.1)$ are made nondimensional according to the scheme (2.42). The propagation characteristics of thermomechanical disturbances in thermoelastic half-spaces for which the relaxation functions are defined by (4.1) have been studied in [14] in detail. We note only that with this choice of relaxation functions, the relaxation coefficients in the expansions $(2.37),(2.38)$ become

$$
\alpha_{i}^{0}=(-1)^{i} \tau^{-(i+1)}, \quad \beta_{i}^{0}=0,
$$

where $\tau$ is the nondimensional thermal relaxation time.

A problem analogous to Problem 1 for thermoelastic half-spaces whose heat conduction obeys the Maxwell-Cattaneo relation has been studied by several authors [22-24] using Laplace transforms. Assuming $\gamma$ is small, they inverted the transforms analytically and illustrated the numerical results graphically. Related numerical results obtained from a ray-series solution have been displayed graphically in [14], 
as well. For purposes of comparison we choose the order of magnitude of thermal parameters to be the same as in the above-mentioned references and display the numerical results for Problem 1 in Figs. 1-6. The Padé extended numerical results are obtained with the techniques detailed in Öncü and Moodie [25]. In all of the figures the ratio of $C_{d}^{2}$ to $C_{s}^{2}$ is chosen as three. This is due to the fact that the isothermal Lamé constants $\lambda$ and $\mu$ are nearly of the same magnitude for most of the materials [26].

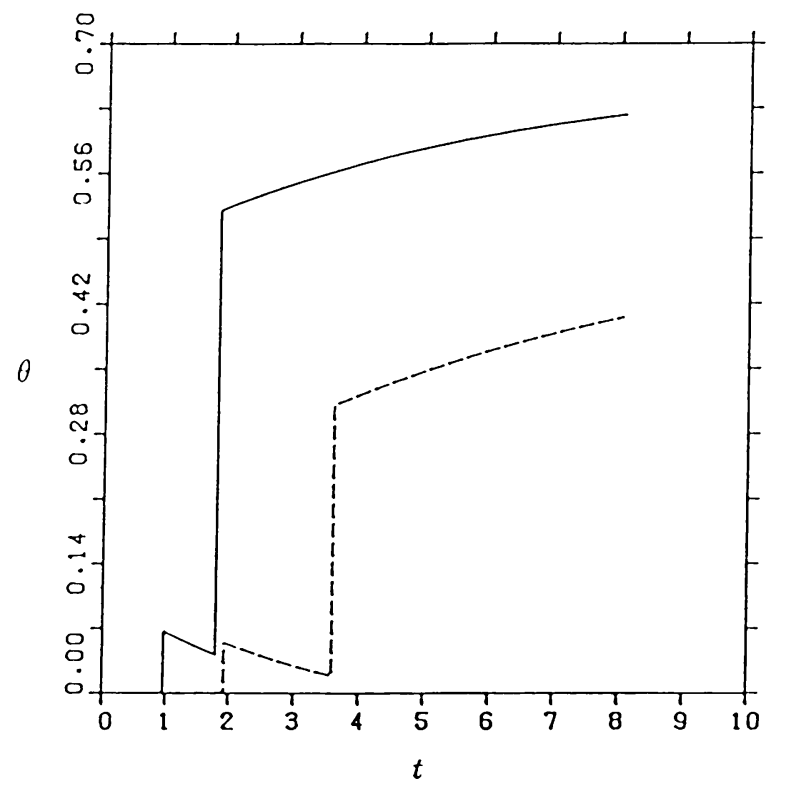

Fig. 1. Variation of nondimension temperature with nondimensional time at $r=2.0(-), r=3.0(--)$ for $C_{d}^{2}=1.0, \tau=3.0, \gamma=0.05$

In Figs. 1 and 2 we plot, respectively, the nondimensional temperature and nondimensional radial stress against nondimensional time for the values of thermoelastic parameters used in [14]. This choice of the thermoelastic parameters is for a material in which quasi-elastic waves propagate faster than quasi-thermal waves. These plots indicate that for a purely thermal boundary disturbance, the discontinuities at the quasi-thermal wavefront decay faster with radial distance than the discontinuities at the quasi-elastic wavefront. This behaviour is more prominent in the case of temperature waves. In Figs. 3 and 4, we depict the influence of the thermoelastic coupling constant on the evolution of discontinuities. It is clear that changes in the thermoelastic coupling constant at small values of $\gamma$ have no significant influence on the response of the medium to purely thermal disturbances. These results are in agreement with the results of the above-mentioned references.

The character change of the fast and slow wavefronts is examined in Figs. 5 and 6. It is seen that whether the quasi-thermal or the quasi-elastic wavefront is faster, the magnitude of the temperature immediately after the arrival of the quasi-thermal wavefront is nearly the same in both cases. For both situations the temperature 


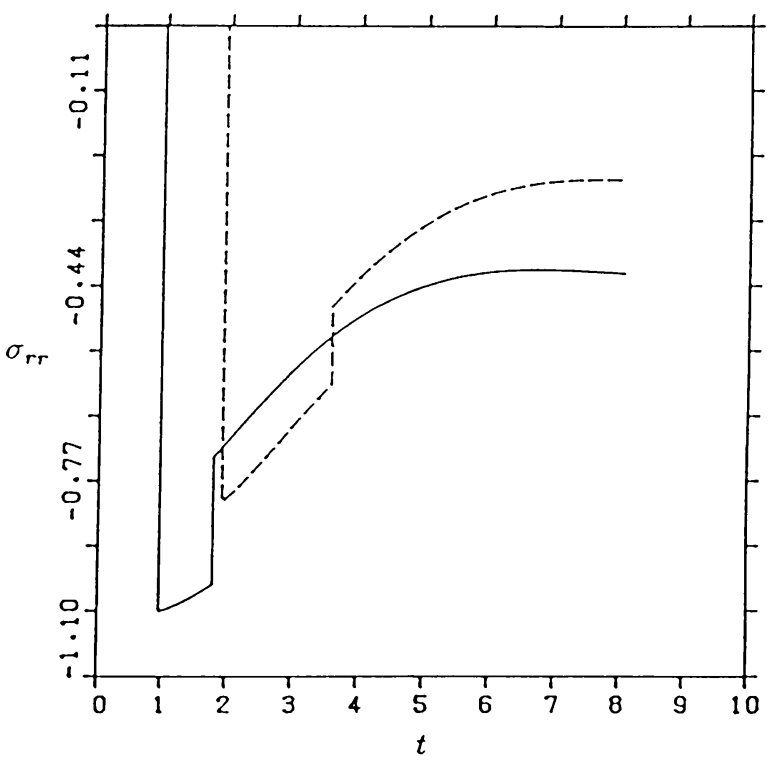

FIG 2. Variation of nondimensional radial stress with nondimensional time at $r=2.0(-), r=3.0(--)$ for $C_{d}^{2}=1.0, \tau=3.0, \gamma=0.05$

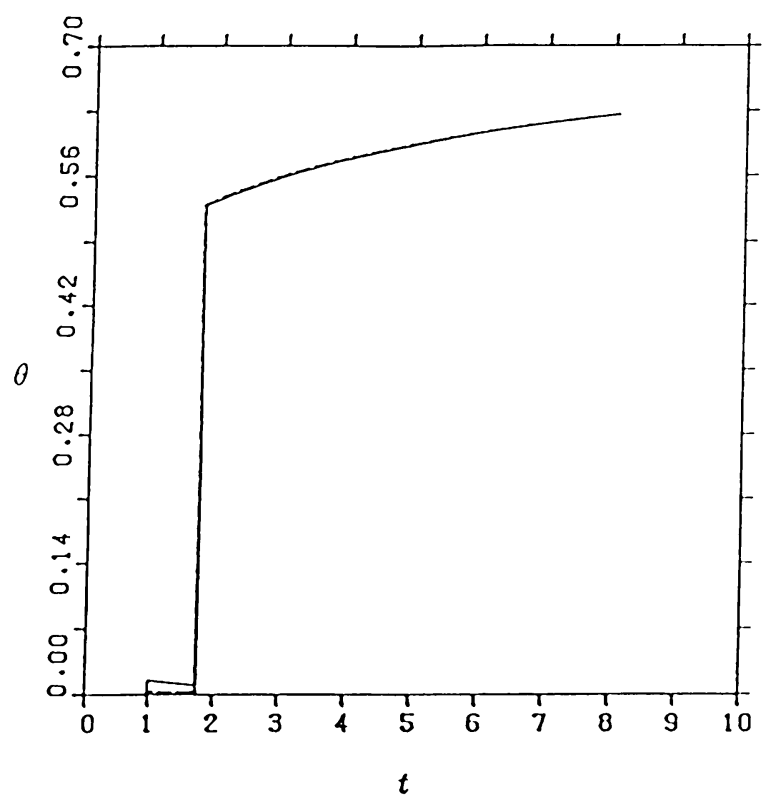

FIG. 3. Variation of nondimensional temperature with nondimensional time at $r=2.0$ for $C_{d}^{2}=1.0, \tau=3.0, \gamma=0.01$ (-), $\gamma=0.001(--)$

response of the material is almost identical after the arrival of the second wavefront. We also see that if the quasi-thermal wavefront is slower then the sign of the jump for the circumferential stress at this wavefront is negative, whereas it is positive in 


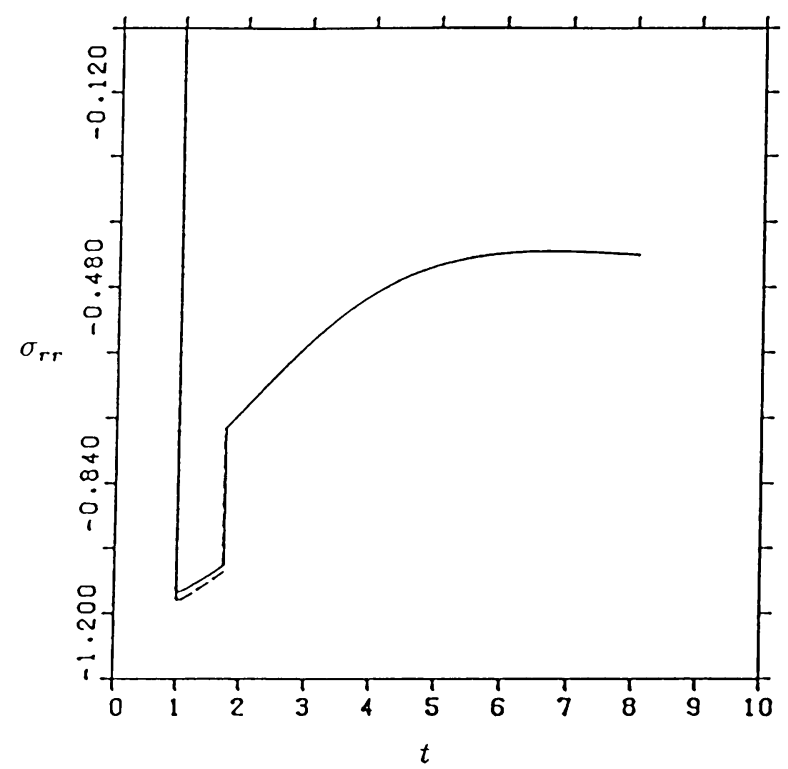

FIG. 4. Variation of nondimensional radial stress with nondimensional time at $r=2.0$ for $C_{d}^{2}=1.0, \tau=3.0, \gamma=0.01 \quad(-)$, $\gamma=0.001(--)$

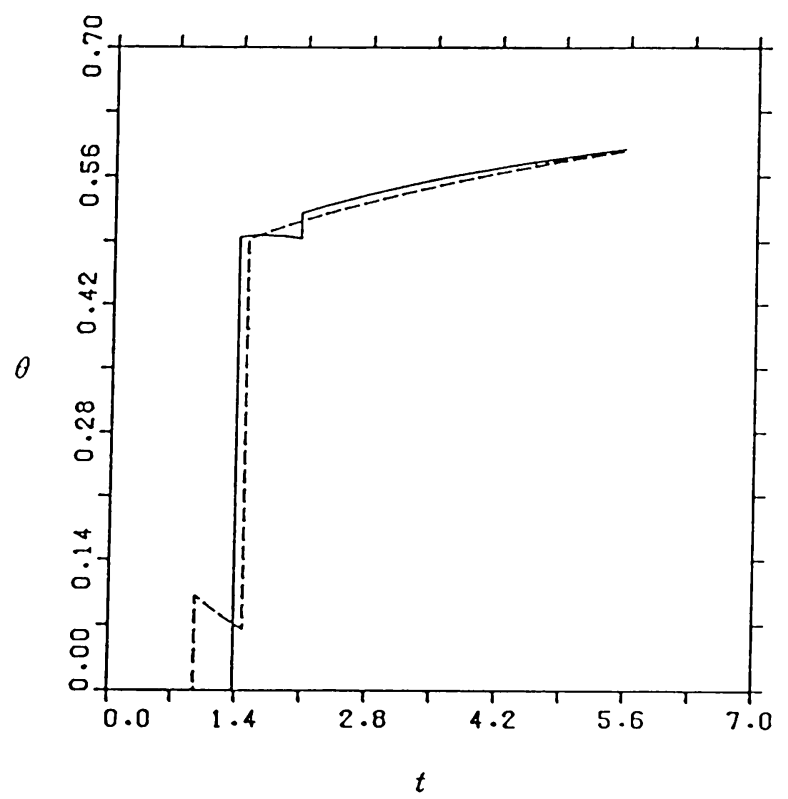

FIG. 5. Variation of nondimensional temperature with nondimensional time at $r=2.0$ for $C_{d}^{2}=0.25(-), C_{d}^{2}=1.0(--)$, $\tau=2.0, \gamma=0.05$ 


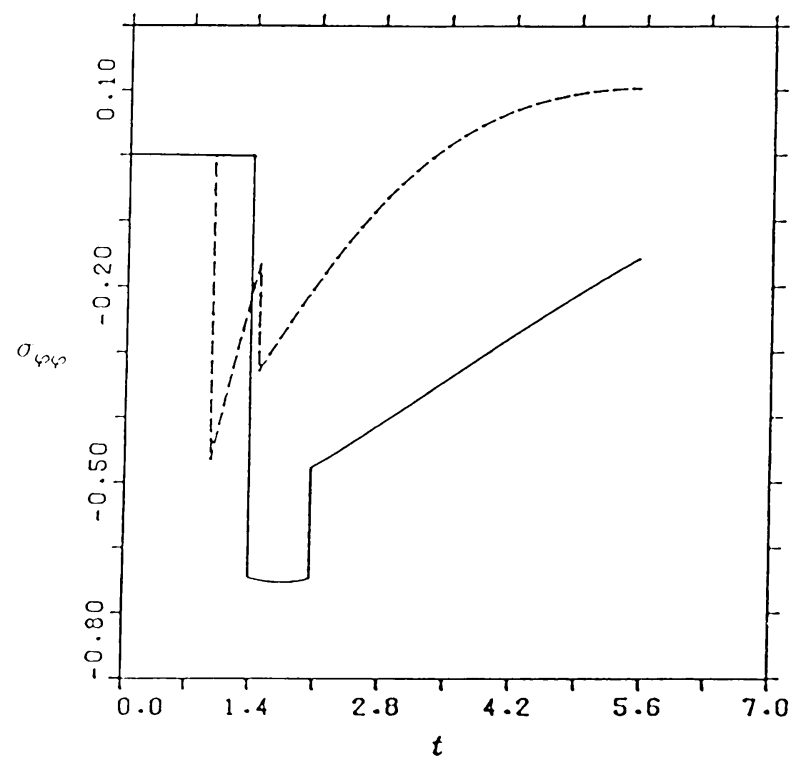

FIG. 6. Variation of nondimensional circumferential stress with nondimensional time at $r=2.0$ for $C_{d}^{2}=0.25(-), C_{d}^{2}=1.00$ $(--), \tau=2.0, \quad \gamma=0.05$

the other case. This result is due to the fact that one of the thermoelastic wavespeeds is very close to $C_{d}$ while the other lies near $\left(\alpha_{0}^{0}\right)^{1 / 2}$. Consequently, if $v_{1}$ is near $C_{d}$ then the condition $v_{1}^{2}>2 C_{s}^{2}$ is most likely to hold and the jump for the circumferential stress at the slower wavefront is positive. If $v_{1}$ lies near $\left(\alpha_{0}^{0}\right)^{1 / 2}$, then both $v_{1}^{2}>2 C_{s}^{2}$ and $v_{1}^{2}<2 C_{s}^{2}$ may happen, although the second condition is expected for most physical situations.

5. Discussion. The theory of Gurtin and Pipkin and of Chen and Gurtin introduces finite speeds for the propagation of thermal transients in rigid materials and eliminates the problem of instantaneous propagation of thermal disturbances. This theory implies two finite speeds of propagation for thermomechanical disturbances in deformable materials. According to this theory, shear waves which generate no volume changes are not affected by thermomechanical coupling. This result is in agreement with that of the thermomechanical theories based on the classical theory of heat conduction. In the case of thermoelastic materials we have shown that when $\alpha_{0}^{0} / C_{d}^{2}<1$, where $\left(\alpha_{0}^{0}\right)^{1 / 2}$ is the velocity of purely thermal and $C_{d}$ the velocity of purely elastic dilatational waves for the material, quasi-elastic waves propagate faster than quasi-thermal waves, whereas when $\alpha_{0}^{0} / C_{d}^{2} \geq 1$ quasi-thermal waves propagate faster than quasi-elastic waves through the medium. For the geometry considered here, we have also shown that the sign of the jump at the slow wavefront of the circumferential stress depends upon the relative magnitudes of $v_{1}$ and $C_{s}$, where $v_{1}$ is the velocity of the slow wavefronts of thermoelastic waves and $C_{s}$ the velocity of shear waves. Other general results obtained here regarding the propagation 
characteristics of disturbances in thermoelastic materials are in agreement with those reported previously by Sawatzky and Moodie [14].

The ray-series approach employed in this paper is a straightforward method for solving the integro-partial differential equations governing the propagation of disturbances in thermoelastic materials and suitable for numerical evaluation of the solution for some distance behind the wavefronts. The Padé-extended ray-series solutions, on the other hand, offer a description of the behaviour of the field variables for larger time intervals after the arrival of the wavefronts. The numerical results presented in this paper are those obtained from the ray series method in conjunction with the use of Padé approximants.

\section{REFERENCES}

[1] C. Cattaneo, Sulla conduzione del calore, Atti Sem. Mat. Fis. Univ. Modena 3, 83-101 (1948)

[2] M. P. Vernotte, Les paradoxes de la théorie continue de l'equation de la chaleur, C.R. Acad. Sci. Paris 246, 3154-3155 (1958)

[3] C. Cattaneo, Sur une forme de l'equation de la chaleur éliminant le paradox d'une propagation instantanée, C.R. Acad. Sci. Paris 247, 431-433 (1958)

[4] M. E. Gurtin and A. C. Pipkin, A general theory of heat conduction with finite wave speeds, Arch. Rat. Mech. Anal. 31, 113-126 (1968)

[5] J. W. Nunziato, On heat conduction in materials with memory, Quart. Appl. Math. 29, 187-204 (1971)

[6] A. E. Green and N. Laws, On the entropy production inequality, Arch. Rational Mech. Anal. 45, 47-53 (1972)

[7] H. W. Lord and Y. Shulman, A generalized dynamical theory of thermoelasticity, J. Mech. Phys. Solids 15, 299-209 (1967)

[8] N. Fox, Generalized thermoelasticity, Internat. J. Engrg. Sci. 7, 437-445 (1969)

[9] P. J. Chen and M. E. Gurtin, On second sound in materials with memory, Z. Angew. Math. Phys. 21, 232-242 (1970)

[10] I. Müller, Die Kältefunktion, eine universelle Funktion der Thermodynamik viskoser wärmeleitender Flüssigkeiten, Arch. Rational Mech. Anal. 40, 1-36 (1971)

[11] I. Müller, The coldness, a universal function in thermoelastic bodies, Arch. Rational Mech. Anal. 41, 319-322 (1971)

[12] A. E. Green and K. A. Lindsay, Thermoelasticity, J. Elasticity 2, 1-7 (1972)

[13] E. S. Şuhubi, Thermoelastic solids, Continuum Physics, Vol II (A. C. Eringen, ed.), Academic Press, New York, 1975, p. 174

[14] R. P. Sawatzky and T. B. Moodie, On thermoelastic transients in a general theory of heat conduction with finite wave speeds, Acta Mech. 56, 165-187 (1985)

[15] B. D. Coleman and W. Noll, The thermodynamics of elastic materials with heat conduction and viscosity, Arch. Rational Mech. Anal. 13, 167-178 (1963)

[16] B. D. Coleman, Thermodynamics of materials with memory, Arch. Rational Mech. Anal. 17, 1-45 (1964)

[17] T. B. Moodie and R. J. Tait, On thermal transients with finite wave speeds, Acta Mech. 50, 97-109 (1983)

[18] M. F. McCarthy, T. B. Moodie, and R. P. Sawatzky, On the propagation of transients through thermoviscoelastic media, Quart. Appl. Math. 46, 539-557 (1988)

[19] J. D. Achenbach, The influence of heat conduction on propagating stress jumps, J. Mech. Phys. Solids 16, 273-282 (1968)

[20] P. Chadwick, Thermoelasticity, the dynamical theory, Progress in Solid Mechanics, Vol. I (J. N. Sneddon and R. Hill, eds.), North-Holland Publishing Company, 1960, p. 265

[21] P. J. Chen and J. W. Nunziato, Thermodynamic restrictions on the relaxation functions of the theory of heat conduction with finite wave speeds, Z. Agnew. Math. Phys. 25, 791-797 (1974)

[22] H. W. Lord and A. A. Lopez, Wave propagation in thermoelastic solids at very low temperature, Acta Mech. 10, 85-98 (1970) 
[23] Y. H. Pao and D. K. Banerjee, Thermal pulses in dielectric crystals, Let. Appl. Engrg. Sci. 1, 33-41 (1973)

[24] A. H. Nayfeh, Propagation of thermoelastic disturbances in non-Fourier solids, AIAA J. 15, 957-960 (1977)

[25] T. S. Öncü and T. B. Moodie, Finite speed thermal transients generated by nonuniform sources applied to circular boundaries in inhomogeneous conductors, Internat. J. Engrg. Sci. 27, 611-621 (1989)

[26] R. J. Atkin and N. Fox, An Introduction to the theory of elasticity, Longman, London and New York, 1980 